\title{
Light, medium-weight or heavy? The nature of the first supermassive black hole seeds
}

\author{
Federica Sassano ${ }^{1,2 \star}$, Raffaella Schneider ${ }^{1,2,3}$, Rosa Valiante ${ }^{2,3}$, Kohei Inayoshi ${ }^{4}$, \\ Sunmyon Chon ${ }^{5}$, Kazuyuki Omukai ${ }^{5}$, Lucio Mayer ${ }^{6}$ and Pedro R. Capelo ${ }^{6}$ \\ ${ }^{1}$ Dipartimento di Fisica, Sapienza, Università di Roma, Piazzale Aldo Moro 5, 00185, Roma, Italy \\ ${ }^{2}$ INFN, Sezione di Roma I, P.le Aldo Moro 2, 00185 Roma, Italy \\ ${ }^{3}$ INAF/Osservatorio Astronomico di Roma, Via di Frascati 33, 00078 Monte Porzio Catone, Italy \\ ${ }^{4}$ Kavli Institute for Astronomy and Astrophysics, Peking University, Beijing 100871, China \\ ${ }^{5}$ Astronomical Institute, Graduate School of Science, Tohoku University, Aoba, Sendai 980-8578, Japan \\ ${ }^{6}$ Center for Theoretical Astrophysics and Cosmology, Institute for Computational Science, University of Zurich, Winterthurerstrasse
} 190, CH-8057, Zürich, Switzerland

Accepted 2021 June 14. Received 2021 June 14; in original form 2020 August 3

\begin{abstract}
Observations of hyper-luminous quasars at $z>6$ reveal the rapid growth of supermassive black holes $\left(\mathrm{SMBHs}>10^{9} \mathrm{M}_{\odot}\right.$ ) whose origin is still difficult to explain. Their progenitors may have formed as remnants of massive, metal free stars (light seeds), via stellar collisions (medium-weight seeds) and/or massive gas clouds direct collapse (heavy seeds). In this work we investigate for the first time the relative role of these three seed populations in the formation of $z>6 \mathrm{SMBHs}$ within an Eddington-limited gas accretion scenario. To this aim, we implement in our semi-analytical dataconstrained model a statistical description of the spatial fluctuations of Lyman-Werner (LW) photodissociating radiation and of metal/dust enrichment. This allows us to set the physical conditions for $\mathrm{BH}$ seeds formation, exploring their relative birth rate in a highly biased region of the Universe at $z>6$. We find that the inclusion of medium-weight seeds does not qualitatively change the growth history of the first SMBHs: although less massive seeds $\left(<10^{3} \mathrm{M}_{\odot}\right)$ form at a higher rate, the mass growth of $\mathrm{a} \sim 10^{9} \mathrm{M}_{\odot} \mathrm{SMBH}$ at $z<15$ is driven by efficient gas accretion (at a sub-Eddington rate) onto its heavy progenitors $\left(10^{5} \mathrm{M}_{\odot}\right)$. This conclusion holds independently of the critical level of LW radiation and even when medium-weight seeds are allowed to form in higher metallicity galaxies, via the so-called super-competitive accretion scenario. Our study suggests that the genealogy of $z \sim 6$ $\mathrm{SMBHs}$ is characterized by a rich variety of $\mathrm{BH}$ progenitors, which represent only a small fraction $(<10-20 \%)$ of all the BHs that seed galaxies at $z>15$.
\end{abstract}

Key words: quasars: supermassive black holes - black hole physics - galaxies: evolution - galaxies: high-redshift

\section{INTRODUCTION}

Starting from their first detection by Fan et al. (2002, 2003), more than 100 supermassive black holes (SMBHs), with masses $\geq 10^{9} \mathrm{M}_{\odot}$, have been observed at $z>6$ as bright quasars (e.g. Willott et al. 2010; Jiang et al. 2016; Bañados et al. 2016; Matsuoka et al. 2019; Yang et al. 2020). The most massive among this high-redshift sample is the hyper-luminous quasar SDSS J0100+2802, with a mass of $1.2 \times 10^{10} \mathrm{M}_{\odot}$ at $z=6.3$ (Wu et al. 2015). The three most distant ones are J0313-1806 (Wang et al. 2021), with a SMBH mass of $1.6 \times 10^{9} \mathrm{M}_{\odot}$, ULAS J1342+0928 (Bañados et al. 2018), with a SMBH mass of $7.8 \times 10^{8} \mathrm{M}_{\odot}$

^ Contact e-mail: federica.sassano@uniroma1.it detected at $z=7.54$, and the recently reported Pōniuā'ena $(\mathrm{J} 1007+2115$, Yang et al. 2020), with a SMBH mass of $1.5 \times 10^{9} \mathrm{M}_{\odot}$ at $z=7.52$, when the Universe was only 0.69 Gyr old. The estimated SMBH masses imply that common mechanisms of Eddington-limited gas accretion onto stellarmass black holes fail to provide a fast enough $\mathrm{BH}$ mass growth at these early cosmic epochs (see, for a recent review, Inayoshi et al. 2019).

A possible scenario is to consider Population III (Pop III) stellar remnants, or light seeds, as progenitors of SMBHs. Pop III stars are expected to form around $z \sim 20-30$ (Bromm 2013), in the first collapsed structures, minihalos with virial temperatures $T_{\text {vir }}<10^{4} \mathrm{~K}$ where the collapse of primordial gas is driven by molecular hydrogen cooling. While hydrodynamical models assuming spherical symmetry 
(Omukai \& Nishi 1998; Omukai et al. 2010) and those starting from cosmological initial conditions (Yoshida et al. 2008) show a remarkable agreement in describing the early collapse phase and the formation of the central hydrostatic core (see also Bromm 2013 and references therein), large uncertainties still remain on the evolution of the post-collapse phase, when disk fragmentation, protostellar evolution, and radiative feedback become important (Omukai \& Palla 2003; Hosokawa et al. 2011; see for a review Greif 2015). The resulting Pop III mass distribution is generally found to be top-heavy, with stellar masses ranging from a few tens to several hundreds of solar masses (Hirano et al. 2014; Hirano et al. 2015; Hosokawa et al. 2016) and a nonnegligible fraction of stars formed in binary or multiple systems (Sugimura et al. 2020). Independent constraints on the Pop III initial mass function (IMF) come from stellar archaeology studies (see Frebel \& Norris 2015 for a thorough review). The low-metallicity tail of the metallicity distribution function of Galactic halo stars and their observed large carbon-to-iron surface abundance ratios appear to be consistent with models where Pop III stars form in the range $[10-300] \mathrm{M}_{\odot}$ with a characteristic mass of $20 \mathrm{M}_{\odot}$ (de Bennassuti et al. 2017) and where the early chemical enrichment is dominated by the explosion of faint supernovae (SNe, Marassi et al. 2014). Even assuming that BH remnants of this first stellar generation can extend to masses of a few 100 s of $\mathbf{M}_{\odot}$, these light seeds can grow in mass to form SMBHs at $z>6$ only if they accrete gas at super-Eddington rates, even in short and intermittent phases (Pezzulli et al. 2016; Pezzulli et al. 2017). Recently, simulations have become capable of studying super-critical accretion in realistic astrophysical environments, assuming slim accretion disks (Sądowski 2009; Madau et al. 2014) or spherically accreting envelopes, without dynamically important angular momentum (Inayoshi et al. 2016; Takeo et al. 2020). The question is whether the high gas densities required to sustain SuperEddington accretion can be found in light seed environments at high redshifts (see for a critical discussion Mayer \& Bonoli 2019).

An alternative scenario to grow SMBHs in the early Universe is to start from heavy seeds, $\sim 10^{5} \mathrm{M}_{\odot} \mathrm{BHs}$ formed from the collapse of supermassive stars (SMSs) (Omukai 2001; Bromm \& Loeb 2003; Wise et al. 2008; Regan \& Haehnelt 2009; Shang et al. 2010; Hosokawa et al. 2012; Latif et al. 2013; Inayoshi et al. 2014; Regan et al. 2014; Becerra et al. 2015; Chon et al. 2016; Latif et al. 2016; Becerra et al. 2018; Wise et al. 2019a; Maio et al. 2019) or in gas-rich galaxy mergers (Mayer et al. 2015; Mayer 2017; Mayer \& Bonoli 2019). In the first case, gas cooling and fragmentation must be avoided to form a single supermassive object. For this reason, heavy seeds are expected to form in pristine halos with virial temperatures $T_{\text {vir }} \geq 10^{4} \mathrm{~K}$, the so-called atomic cooling halos, where fragmentation is avoided because of the lack of metals and molecular hydrogen cooling is suppressed by a sufficiently strong radiation field in the Lyman-Werner (LW) band (Omukai et al. 2008; Dijkstra et al. 2014; Sugimura et al. 2014; Wolcott-Green et al. 2017; Wolcott-Green et al. 2020) or by dynamical heating caused by rapidly growing dark matter halos (Mayer et al. 2015; Wise et al. 2019a). In addition to avoiding fragmentation, very high accretion rates, with $\dot{M}>0.01 \mathrm{M}_{\odot} \mathrm{yr}^{-1}$, are required to reduce radiative feed- back from the growing protostar onto the accretion flow (Hosokawa et al. 2012; Chon et al. 2018; Matsukoba et al. 2019).

Finally, a third scenario foresees the formation of $\mathrm{BH}$ seeds with mass $\sim 10^{3} \mathrm{M}_{\odot}$, also called Intermediate Mass Black Holes (IMBHs), that we will refer to as medium-weight seeds. Their formation is expected to occur through runaway stellar collisions in dense star clusters (Omukai et al. 2008; Volonteri 2010; Davies et al. 2011; Devecchi et al. 2012; Katz et al. 2015; Sakurai et al. 2017; Stone et al. 2017; Reinoso et al. 2018; Tagawa et al. 2020). These are supposed to originate in atomic cooling halos where the gas initially undergoes an almost isothermal collapse, similar to the early formation phase of heavy seeds. Hence, their formation requires conditions similar to the ones discussed above, lack of metal fine structure line and $\mathrm{H}_{2}$ cooling. Howeyer, a dense star cluster may form provided that dust cooling drives fragmentation in the late phase of the collapse (Omukar et al. 2008). It is important to stress that this simple picture may be significantly affected by the dynamics of the infalling gas. Indeed, Chon \& Omukai (2020) show that, in the absence of $\mathrm{H}_{2}$ cooling, although dust cooling promotes the formation of a few thousand low-mass stars, the strong accreting flow preferentially feeds the central star which grows supermassive. Hence, in this super competitive accretion (SCA) mode, the formation of heavy seeds may continue even in moderately enriched halos, and medium-weight seeds form only when metal line cooling starts to decrease the gas temperature, at $Z \sim 10^{-3} Z_{\odot}$, lowering the accretion rate.

The importance of medium-weight seeds is not only related to SMBH genealogy, but has a number of additional implications in astrophysics. Medium-weight seeds would extend the correlation between the stellar and nuclear black hole masses to dwarf galaxies (Greene et al. 2010: Safonova \& Shastri 2010; Reines \& Volonteri 2015; Mezcua et al. 2018), they could explain the existence of Ultra Luminous X-ray sources (Miller et al. 2004; Fritze et al. 2018; Shen 2019; Barrows et al. 2019; Baldassare et al. 2020), and are necessary to prevent tidal distruption events in young star clusters observed in the galactic nucleus (Kim et al. 2004). These $\sim 10^{3} \mathrm{M}_{\odot}$ $\mathrm{BHs}$ will be targeted by future third-generation gravitational telescopes, such as the Laser Interferometer Space Antenna (LISA) (Coleman Miller \& Hamilton 2002; Sesana et al. 2005; Amaro-Seoane \& Santamaria 2010; Kremer et al. 2019), but recent observations (Ballone et al. 2018; Argo et al. 2018; Takekawa et al. 2019; Nguyen et al. 2019; Woo et al. 2019; Barack et al. 2019) have found evidence of their existence in the Local Universe.

In this work, we aim at assessing the relative importance of light, medium-weight and heavy seeds, in the mass growth histories of $z \sim 6$ SMBHs. To this aim, we have largely improved our original seeding prescription described in Valiante et al. (2016b) and Valiante et al. (2018a) in order to investigate the formation sites of all these three families of $\mathrm{BH}$ seeds in the cosmological evolution of a typical $z \sim 6 \mathrm{SMBH}$ and its host galaxy. In particular, we present here a more physical treatment of the inhomogeneous radiative and chemical feedback. We quantify the statistical distributions of all $\mathrm{BH}$ seeds in several independent simulations of the final SMBH, and explore how these are impacted by 
assuming different conditions (critical LW flux, metallicity, and dust-to-gas mass ratios) for their formation.

The paper is organized as follows. In section 2 we briefly describe the semi-analytical model adopted in our analysis. Section 3 presents the new features implemented for this work, i.e. the medium-weight seed formation scenario (sections 3.1 and 3.2) and the inhomogeneous treatment of metal enrichment and radiative LW flux (section 3.3). In section 4 , we describe the main results, that will be discussed and summarized in sections 5 and 6 .

For our analysis, we will assume a Planck Cosmology with $\Omega_{m}=0.314, \Omega_{\Lambda}=0.686, n_{s}=0.96, h=0.674$ and $\sigma_{8}=0.834$ from Ade et al. (2014).

\section{DESCRIPTION OF THE MODEL}

Here we summarize the main features of the semi-analytical, data constrained model, GAMETE/QSOdust (GQd) developed to simulate the cosmological build-up of a $z \sim 6 \mathrm{SMBH}$ and its host galaxy (Valiante et al. 2011, 2014, 2016b).

GQd follows the co-evolution of nuclear BHs and their host galaxies and, at the same time, the metal and dust enrichment of their interstellar medium (ISM), in a cosmological context. For this reason, GQd is suitable to investigate the birth environments of $\mathrm{BH}$ seeds in high-redshift galaxies, their subsequent mass growth through gas accretion and mergers, and their relative contribution to the final SMBH mass at $z \sim 6$. We refer the reader to Valiante et al. (2016b) (and references therein) for a detailed description of the original model.

\subsection{Dark matter halos evolution}

For a statistical analysis we reconstruct ten merger histories of a dark matter $(\mathrm{DM})$ halo of $M_{\text {halo }}=10^{13} \mathrm{M}_{\odot}$ at $z_{0}=6.42$, presumably hosting a SMBH (Willott et al. 2003; Cubbon et al. 2007). We perform a binary Monte Carlo algorithm to decompose, backward in time, the massive DM halo into its progressively less massive progenitors, from $z_{0}$ back to $z=24$.

At a given redshift $z$, the resolution mass of the simulated merger trees, i.e. the minimum mass of a virialized structure, is described as in Valiante et al. (2020):

$$
M_{\text {res }}(z)=10^{-3} M_{\text {halo }}\left(\frac{1+z}{1+z_{0}}\right)^{\beta}
$$

where $\beta=-7.5$ (Valiante ef al. 2016b), so that $M_{\text {res }} \sim$ $10^{6}\left(10^{10}\right) \mathrm{M}_{\odot}$ at $z=24\left(z=z_{0}\right)$. Lower mass fragments, i.e. unresolved structures with $M<M_{\text {res }}$, account for the external medium from which halos accrete mass, that we refer to as intergalactic medium (IGM). The functional form in Eq. 2.1 and the redshift-dependent characteristic time interval, $\Delta t$ (ranging between 0.2 and $2 \mathrm{Myr}$ ), are designed to ensure the binarity of the merger tree $(<2$ progenitors per halo), to resolve the formation of high- $z$ minihalos, to reproduce the halo mass function predicted by the Extended Press-Schechter (EPS) formalism (Lacey \& Cole 1993) and to contain computational costs.

\subsection{Star formation and feedback}

\subsubsection{Star formation law}

Along a merger tree, each progenitor galaxy can form stars according to the available gas budget, $M_{\text {gas }}$. The star formation rate (SFR) is described as:

$S F R=f_{\text {cool }} M_{\text {gas }} \epsilon / \tau_{\text {dyn }}(z)$,

where $\tau_{\text {dyn }}(z)$ is the halo dynamical time, $\epsilon=\epsilon_{\text {quiesc }}+\epsilon_{\text {burst }}$ is the sum of the quiescent and merger-driven starburst efficiencies and is one of the model free parameters, that have been set to reproduce the observed properties of a prototypical $z \sim 6$ QSO and its host galaxy (see Table 1 for the complete set of free parameters). We assume stars to form with a constant efficiency $\epsilon_{\text {quiesc }}=0.1$ that can be enhanced to $\epsilon_{\text {quiesc }}+\epsilon_{\text {burst }}$ during galaxy major mergers due to a reduction in the timescales for star formation from $\tau_{\text {quies }}=\tau_{\text {dyn }} / \epsilon_{\text {quiesc }}$ to $\tau_{\text {burst }}=\tau_{\text {dyn }} /\left(\epsilon_{\text {burst }}+\epsilon_{\text {quiesc }}\right)$. Following Valiante et al. (2011), we assume $\epsilon_{\text {burst }}$ to be a function of the ratio $\mu$ between the mass of the less massive halo over the more massive companion and to be described by a normalized Gaussian distribution:

$\epsilon_{\text {burst }}=\frac{e^{-\left(\mu-\mu_{\text {crit }}\right)^{2} / 2 \sigma_{\text {burst }}^{2}}}{\sqrt{2 \pi} \sigma_{\text {burst }}}$

with $\sigma_{\text {burst }}=0.05, \mu_{\text {crit }}=1$, and $\mu>1 / 4$, which defines the threshold for galaxy major mergers. For equal mass mergers $\left(\mu=\mu_{\text {crit }}\right)$, this leads to a maximum value of $\epsilon_{\text {burst }}=8$, reducing the timescale for star formation, $\tau_{\text {burst }} / \tau_{\text {quies }}=\epsilon_{\text {quiesc }} /\left(\epsilon_{\text {quiesc }}+\epsilon_{\text {burst }}\right) \sim 0.012$.

Finally, the parameter $f_{\text {cool }}$ in Eq. 2.2 quantifies the reduced cooling efficiency of minihalos with respect to atomic cooling halos, that we discuss below.

\subsubsection{Radiative feedback}

In each galaxy, the efficiency of gas cooling and star formation is regulated by radiative feedback. In our model we account for the effects of both photo-dissociating and photoheating feedback.

At low metallicity, the gas cooling efficiency in minihalos relies only on molecular hydrogen, that can be easily dissociated by photons in the LW band. We account for this effect through the parameter $f_{\text {cool }}$ entering in Eq. 2.2. In particular, we set $f_{\text {cool }}=1$ in atomic cooling halos, whereas in minihalos its value $(\leq 1)$ depends on the halo virial temperature, $T_{\mathrm{vir}}$, redshift, gas metallicity, and intensity of the illuminating $\mathrm{LW}$ flux, $J_{\mathrm{LW}}$, expressed in units of $10^{-21} \mathrm{erg} / \mathrm{s} / \mathrm{Hz} / \mathrm{cm}^{2} / \mathrm{sr}$ (see Valiante et al. 2016b; de Bennassuti et al. 2017, for details). In Fig. 1 we show the redshift evolution of the reduced cooling efficiency for the least massive halos (for halos with $M_{\mathrm{vir}}=M_{\text {res }}(z)$ ) assuming three different values of the LW irradiating flux, $J_{\mathrm{LW}}=0,1$, and 10 , and a constant gas metallicity equal to $Z=0$. Similar results are found for gas metallicity in the range $0 \leq Z \leq 10^{-2} Z_{\odot}$. The figure shows that even when $J_{\mathrm{LW}}=0, f_{\text {cool }}$ ranges between 0.02 to 1 due to less efficient cooling by molecular hydrogen. At $z>12, M_{\text {res }}(z)$ corresponds to mini-halos and $f_{\text {cool }}$ is very sensitive to the illuminating LW radiation field. At $z \leq 12, M_{\text {res }}(z)$ grows above the halo mass with $T_{\mathrm{vir}}=10^{4} \mathrm{~K}$ and $f_{\text {cool }}=1$ in atomic cooling halos. When $J_{\mathrm{LW}}=100, f_{\text {cool }}=0$ at all redshifts greater 


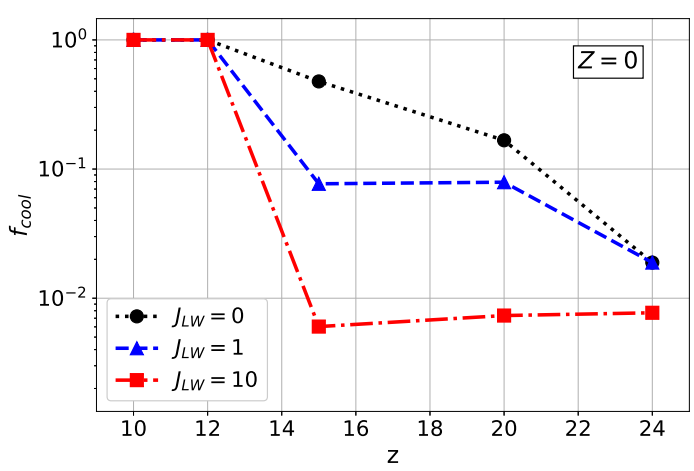

Figure 1. The predicted reduced cooling efficiency $f_{\text {cool }}$ in minihalos as a function of redshift. The black, blue, and red dashed lines correspond to different values of the LW flux $\left(J_{\mathrm{LW}}=0,1,10\right.$, respectively) illuminating halos with mass equal to the resolution mass of the simulation, $M_{\mathrm{vir}}=M_{\mathrm{res}}(z)$, and with a constant gas metallicity $Z=0$. The resulting cooling efficiency is independent of metallicity for poorly enriched $\left(Z \leq 10^{-2} Z_{\odot}\right)$ minihalos.

than 12 and star formation in mini-halos is completely suppressed.

Even in atomic cooling halos star formation can be inhibited as a consequence of the increased gas temperature within photo-ionized regions. We assume $\epsilon=0$ in Eq. 2.2 when the halo virial temperature is below the IGM temperature, $T_{\mathrm{vir}}<T_{\mathrm{IGM}}$. The latter is computed as:

$T_{\mathrm{IGM}}=Q_{\mathrm{HII}}(z) T_{\text {reio }}+\left[1-Q_{\mathrm{HII}}(z)\right] T_{\mathrm{gas}}$

where $Q_{\mathrm{HII}}(z)$ is the filling factor of HII regions, $T_{\text {reio }}=2 \times 10^{4}$ $\mathrm{K}$ is the post-reionization temperature and $T_{\text {gas }}=170 \mathrm{~K}$ $[(1+z) / 100]^{2}$. We compute the time evolution of $Q_{\mathrm{HII}}(z)$ as:

$\frac{d Q_{\mathrm{HII}}}{d t}=f_{\mathrm{esc}} \dot{n}_{\gamma} / n_{\mathrm{H}}-\alpha_{B} C n_{\mathrm{H}}(1+z)^{3} Q_{\mathrm{HII}}$

where $f_{\text {esc }}=0.1$ is the escape fraction of ionizing photons, $\dot{n}_{\gamma}$ is the total production rate of ionizing photons per unit volume summed over all the emitting sources, $n_{\mathrm{H}}$ is the comoving hydrogen number density in the IGM, $\alpha_{B}=2.6 \times 10^{-13}$ $\mathrm{cm}^{3} \mathrm{~s}^{-1}$ is the hydrogen recombination rate and $C=3$ is the clumping factor (for more details, see Valiante et al. 2016b).

\subsubsection{Chemical and mechanical feedback}

In GQd stars form according to a Larson IMF (Larson 1998): $\phi\left(m_{*}\right) \propto m_{*}^{(\alpha-1)} e^{-m_{\mathrm{ch}} / m_{*}}$

where $m_{*}$ is the stellar mass $/ m_{\mathrm{ch}}$ is the characteristic mass and $\alpha=-1.35$. In metal free/poor $\left(Z<Z_{\mathrm{cr}}=10^{-3.8} Z_{\odot}\right)$ environments we-adopt an IMF constrained by stellar archaeology data (de Bénnássuti et al. 2014), with Pop III stars forming in the mass range $[10-300] \mathrm{M}_{\odot}$ and $m_{\mathrm{ch}}=20 \mathrm{M}_{\odot}$. In addition, when the star formation efficiency is low, we randomly sample the above IMF to predict the stellar mass distribution present in each system (see Valiante et al. 2016b, for details). Once the metallicity increases above the threshold $Z>Z_{\mathrm{cr}}=10^{-3.8} Z_{\odot}$, metal fine-structure line cooling becomes efficient (Omukai et al. 2005) and we assume Pop II/I stars to form in the range $[0.1-100] \mathrm{M}_{\odot}$ with $m_{\mathrm{ch}}=0.35 \mathrm{M}_{\odot}$.
Table 1. Free parameters of the Reference and SCA models. $\epsilon_{\text {quiesc }}$ is the star formation efficiency (see Eq. 2.2), $\epsilon_{\mathrm{w}, \mathrm{SN}}$ is the SNdriven wind efficiency (see Eq. 2.7), $\alpha_{\mathrm{BH}}$ quantifies the efficiency of $\mathrm{BH}$ accretion (see Eq. 2.9) and $\boldsymbol{\epsilon}_{\mathrm{w}, \mathrm{AGN}}$ is the BH-driven wind efficiency (see Eq.2.11). For each set of models, these values have been calibrated to reproduce the observed properties of the $z=$ 6.42 QSO J1148 and its host galaxy (see Valiante et al. 2016b).

\begin{tabular}{ccccc}
\hline Model & $\epsilon_{\text {quiesc }}$ & $\epsilon_{\mathrm{w}, \mathrm{SN}}$ & $\alpha_{\mathrm{BH}}$ & $\epsilon_{\mathrm{w}, \mathrm{AGN}}$ \\
\hline Reference & 0.1 & $1.6 \cdot 10^{-3}$ & 150 & $2.5 \cdot 10^{-3}$ \\
SCA & 0.1 & $1.6 \cdot 10^{-3}$ & 80 & $2.5 \cdot 10^{-3}$ \\
\hline
\end{tabular}

In each galaxy, evolving stars progressively enrich the ISM with metals and dust. Their abundances are computed adopting the grids of stellar yields provided by van den Hoek \& Groenewegen (1997) and Zhukovska et al. (2008) for AGB stars $\left(1-8 \mathrm{M}_{\odot}\right)$, Woosley \& Weaver (1995) and Bianchi et al. (2009) for core-collapse SNe $\left(10-40 \mathrm{M}_{\odot}\right)$ and Heger \& Woosley (2002) and Bianchi et al? (2009) for pair-instability SNe (PISNe, 140-260 M०). For our adopted Pop III IMF, the largest contribution to the metal and dust yields is provided by the most massive stars (see the left panel of Fig. 2 in de Bennassuti et al. 2014), for which the assumption of instantaneous recycling is a good approximation. Hence, Pop III stars are assumed to evolve instantaneously, i.e. their lifetime is the characteristic timeinterval of the merger tree, while Pop II/I stellar lifetimes are computed according to the parametric form proposed by Raiteri et al. (1996) and depend on the stellar mass and metallicity. Once injected in the ISM, the abundance of dust grains is computed accounting for grain destruction by $\mathrm{SN}$ shocks and grain growth in the dense, cold ISM phase (for additional details, see Valiante et al. 2014).

Finally, SN explosions deposit energy in the ISM, driving a galaxy-scale wind (mechanical feedback),

$\frac{d M_{\mathrm{ej}, \mathrm{SN}}(t)}{d t}=\frac{2 E_{\mathrm{SN}} \epsilon_{\mathrm{w}, \mathrm{SN}} R_{\mathrm{SN}}(t)}{v_{\mathrm{e}}^{2}}$

that is proportional to the rate of $\mathrm{SN}$ explosions, $R_{\mathrm{SN}}(t)$, to the average $\mathrm{SN}$ explosion energy, $E_{\mathrm{SN}}$, and to an efficiency parameter, $\epsilon_{\mathrm{w}, \mathrm{SN}}$, that accounts for the fact that only a small fraction of the total energy injected by the $\mathrm{SNe}$ is in kinetic form and coupled to the gas, while the rest is dissipated and converted into thermal energy (see e.g. Walch \& Naab 2015). The quantity $R_{\mathrm{SN}}(t)$ depends on the SFR and on the stellar IMF, $E_{\mathrm{SN}}=2.7 \cdot 10^{52} \mathrm{erg}$ for Pop III stars and $1.2 \cdot 10^{51}$ erg for Pop II/I stars, and $\epsilon_{\mathrm{w}, \mathrm{SN}}$ is a free parameter that we calibrate based on the observations (see Table 1).

\section{3 $\quad$ BH seeding prescriptions}

As introduced in section 1, seeds expected to form under specific environmental conditions (level of illuminating LW radiation, metal and dust composition of the ISM). In GQd such properties are computed consistently with the overall evolution of the galaxy and nuclear black hole populations (Valiante et al. 2016b; Valiante et al. 2018a,b).

Light seeds form as end-products of Pop III stars, in minihalos or atomic cooling halos where gas cooling is dominated by $\mathrm{H}_{2}$. This implies that the illuminating LW flux 
must be sub-critical, $J_{\mathrm{LW}}<J_{\mathrm{cr}}$, and that metal- and dustcooling must not operate, hence the metallicity $Z<Z_{\text {cr }}$ and the gas-to-dust mass ratio $\mathcal{D}<\mathcal{D}_{\text {cr. }}$. In these conditions, the star formation efficiency is relatively low and only a small number of stars is formed. The mass of the BH remnants depends on the initial mass of the (stochastically sampled) stars (Woosley et al. 2002): stars in the mass ranges $[40-140] \mathrm{M}_{\odot}$ and [260 - 300] $\mathrm{M}_{\odot}$ directly collapse into a $\mathrm{BH}$ with a mass equal to the progenitor mass; in the mass range $[140-260] \mathrm{M}_{\odot}$, the stars explode as Pair Instability SN (PISN) and leave no remnant. Depending on the star formation efficiency, these different mass ranges are unequally sampled and therefore the emerging $\mathrm{BH}$ mass distribution is not unique.

We assume that the heaviest among all the formed BHs migrate to the centre by dynamical friction and we tag this as the nuclear light seed. If a halo hosts multiple Pop III star formation episodes, we repeat the random sampling procedure and we assume the heaviest among the new $\mathrm{BH}$ population to migrate to the centre and to merge with the previous nuclear $\mathrm{BH}$, generating a new heavier light seed.

Heavy seeds mediated by the collapse of a SMS require the gas to monolithically collapse with no fragmentation (Omukai et al. 2008). This, in turn, requires the gas to be hosted in atomic cooling halos that are illuminated by a sufficiently strong LW flux to inhibit $\mathrm{H}_{2}$ cooling and, similarly to light seeds, metal and dust cooling must not operate. In this scenario we plant a heavy seed of $10^{5} \mathrm{M}_{\odot}$ in atomic cooling halos where $J \geq J_{\mathrm{cr}}$, and $Z<Z_{\mathrm{cr}}$ and $\mathcal{D}<\mathcal{D}_{\text {cr. }}$. In addition, we require the halo not to have experienced previous episodes of star formation and to have a sufficiently large reservoir of gas $M_{\text {gas }} \geq 10^{6} \mathrm{M}_{\odot}$ (condition required to fuel strong accretion onto the forming SMS).

Following Valiante et al. (2016b), in our reference model (that we call R300) we assume $J_{\mathrm{cr}}=300$ (expressed in units of $\left.10^{-21} \mathrm{erg} / \mathrm{s} / \mathrm{Hz} / \mathrm{cm}^{2} / \mathrm{sr}\right), Z_{\mathrm{cr}}=10^{-3.8} Z_{\odot}$, and $\mathcal{D}_{\text {cr }}=4.4 \cdot 10^{-9}$ (Schneider et al. 2012).

\subsection{Black hole growth and feedback}

The model GQd follows the mass growth of nuclear black holes through gas accretion and the coalescence with other black holes, starting from a seed population that depends on the environmental conditions as discussed above.

We assume that two nuclear BHs coalesce only in major merger events, i.e. when $\mu \geq 1 / 41$ Although post-merger $\mathrm{BH}$ ejection due to asymmetric $\mathrm{GW}$ emission may have an impact on the SMBH growth, especially at high z (see e.g. Pezzulli et al. 2016), in the present analysis we neglect the gravitational recoil effect in other to compare our results with Valiante et al. (2016b) but focusing on the relative role of all three $\mathrm{BH}$ seeds populations for $z>6 \mathrm{SMBHs}$ growth.

In minor halo-halo mergers $(\mu<1 / 4)$ we instead assume that only the most massive $\mathrm{BH}$ will settle in the nuclear region of the newly formed galaxy, where it can start accreting

1 We assume a galaxy merger to occur within the redshiftdependent timestep of the simulation, $\Delta t$, which ranges from a fraction to a few Myr. gas. The lighter one is considered as a satellite, wandering in the galaxy outskirts, and we do not follow its subsequent evolution. Such a conservative assumption is independent of the mass ratio of the two BHs involved in the galaxy merger event. However, during wet (gas-rich) minor galaxy mergers (down to 1:10), the satellite $\mathrm{BH}$ may grow faster than the primary so that it may orbit towards the centre as well (see e.g. Callegari et al. 2009, 2011; Van Wassenhove et al. 2014; Capelo et al. 2015). We plan to investigate this effect, together with a more refined description of $\mathrm{BH}$ dynamics (including gravitational recoil) in future works.

Thus, not all the nuclear BHs present at $z>6.4$ will directly contribute to the final $\mathrm{SMBH}$ mass, as some of these may be involved in minor halo-halo mergers and become wandering $\mathrm{BHs}$ at later times. The real SMBH progenitors are identified by reconstructing the assembly history backwards in time up to the seeds formation epoch.

As in Valiante et al. (2011, 2014, 2016a), BH mass growth by gas accretion proceeds at a rate given by $\dot{M}_{\mathrm{acc}}=\min \left[\dot{M}_{\mathrm{Edd}}, \dot{M}_{\mathrm{BHL}}\right]$, where $\dot{M}_{\mathrm{Edd}}$ is the Eddington rate,

$\dot{M}_{\mathrm{Edd}}=\frac{4 \pi G M_{\mathrm{BH}} m_{\mathrm{p}}}{\epsilon_{\mathrm{r}} \sigma_{\mathrm{T}} c}$,

and $\dot{M}_{\mathrm{BHL}}$ is the Bondi-Hoyle-Lyttleton accretion rate (Hoyle \& Lyttleton 1939; Bondi \& Hoyle 1944; Bondi 1952),

$\dot{M}_{\mathrm{BHL}}=\frac{4 \pi \alpha_{\mathrm{BH}} G^{2} M_{\mathrm{BH}}^{2} \rho_{\mathrm{gas}}\left(r_{\mathrm{A}}\right)}{c_{\mathrm{s}}^{3} y}$.

In the above equations, $m_{\mathrm{p}}$ is the proton mass, $\epsilon_{\mathrm{r}}=0.1$ is the radiative efficiency, $\sigma_{\mathrm{T}}$ is the Thomson scattering crosssection, $G$ is the gravitational constant, $c$ is the speed of light, $\rho_{\text {gas }}\left(r_{\mathrm{A}}\right)$ is the gas density ${ }^{2}$ at the radius of gravitational influence of the $\mathrm{BH}$ (also called Bondi radius, $r_{\mathrm{A}}=2 G M_{\mathrm{BH}} / c_{\mathrm{s}}^{2}$ ), and $c_{\mathrm{s}}$ is the sound speed. The dimensionless parameter $\alpha_{\mathrm{BH}}$ is usually introduced in numerical simulations to compensate for the lack of resolution that heavily underestimates the BHL accretion rate (Springel et al. 2005) and is a free parameter of the model (see Table 1).

Numerical simulations show that feedback due to $\mathrm{SNe}$ appears crucial in regulating $\mathrm{BH}$ growth in low-mass galaxies (Dubois et al. 2015) and therefore affects the $\mathrm{BH}$ mass distribution after the formation epoch of $\mathrm{BH}$ seeds (Habouzit et al. 2016a). Our model describes the total outflow rate from a galaxy as the sum of the active galactic nucleus (AGN) and SN contributions,

$\frac{d M_{\mathrm{ej}}(t)}{d t}=\frac{d M_{\mathrm{ej}, \mathrm{AGN}}}{d t}+\frac{d M_{\mathrm{ej}, \mathrm{SN}}}{d t}$,

and the energy-driven AGN gas outflow (wind) is described as:

$\frac{d M_{\mathrm{ej}, \mathrm{AGN}}}{d t}=2 \epsilon_{\mathrm{w}, \mathrm{AGN}} \epsilon_{\mathrm{r}} \dot{M}_{\mathrm{accr}}\left(\frac{c}{v_{\mathrm{e}}}\right)^{2}$,

2 Following Valiante et al. (2011), we assume the gas to follow an isothermal density distribution with a flat core. The core radius is assumed to be 0.012 times the dark matter halo virial radius and the profile is normalized such that at each time the total gas mass predicted by the model is enclosed within the virial radius. We refer the reader to Valiante et al. (2011) for additional details. 
where $\epsilon_{\mathrm{W}, \mathrm{AGN}}$ is the wind efficiency. AGN winds are assumed to be in action when the accretion rate exceeds $10^{-2} \dot{M}_{\text {Edd }}$ $\left(d M_{\mathrm{ej}, \mathrm{AGN}} / d t=0\right.$ otherwise). Even with our simplified treatment of AGN and SN feedback, BHs often starve due to gas evacuation, particularly in the small halos at high- $z$ that are characterized by small escape velocities. In this regime, the $\mathrm{BH}$ duty-cycle is set by halo mass growth via mergers, that provide additional gas supply to the nuclear regions, restarting a new cycle of star formation and $\mathrm{BH}$ growth.

\section{NEW FEATURES OF THE MODEL}

In this section we describe the new physically motivated prescriptions implemented in the model to investigate the occurrence of light, heavy and medium-weight seeds in a cosmological context and their relative importance/role in the formation of $z>6$ SMBHs.

\subsection{Medium-weight seeds}

As we have seen in section 2.3, a necessary condition for the monolithic collapse is to avoid fragmentation. When dust-driven cooling is effective, that is when $\mathcal{D} \geq \mathcal{D}_{\text {cr }}$ (Schneider et al. 2012), fragmentation occurs at very high densities and in a very compact region, potentially leading to the formation of a dense stellar cluster (Omukai et al. 2008; Clark et al. 2008).

The question is then to understand what is the final fate of this system. During their lifetime, collisional stellar systems evolve as a result of dynamical interactions. In an equal-mass system, the central cluster core initially contracts as the system attempts to reach a state of thermal equilibrium: energy conservation leads to a decrease in the core radius as evaporation of the less bound stars proceeds. As a result, the central density increases and the central relaxation time decreases. The core then decouples thermally from the outer region of the cluster. Core collapse then speeds up as it is driven by energy transfer from the central denser region (Gürkan et al. 2004; Reinoso et al. 2018; Reinoso et al. 2019). This phenomenon is greatly enhanced in multi-mass systems like realistic star clusters. In this case, the gravothermal collapse happens on a shorter time-scale as dynamical friction causes the more massive than $m_{*}$ stars to segregate in the center on a timescale $t_{\mathrm{df}}=\left(\langle m\rangle / m_{*}\right) t_{\mathrm{rh}}$, where $t_{\mathrm{rh}}$ is the half mass relaxation time-scale, and $\langle m\rangle$ is the mean stellar mass in the cluster (Portegies Zwart \& McMillan 2002; Devecchi \& Volonteri 2009). If mass segregation sets in before the more massive stars evolve out of the main sequence $(\sim 3 \mathrm{Myr})$, then a subsystem decoupled from the rest of the cluster can form, where star-star collisions can take place in a runaway fashion, ultimately leading to the growth of a very massive star (VMS). The upper limit on the duration of the process ( $\sim 3 \mathrm{Myr})$ is set by the requirement to avoid the explosion of the SNe, which would sweep away the system (Sakurai et al. 2017).

If a VMS forms above $260 \mathrm{M}_{\odot}$, it would directly collapse into a $\mathrm{BH}$ of comparable mass. In addition, the $\mathrm{BH}$ can still gain mass by accretion of stars and from the surrounding gas (Devecchi \& Volonteri 2009). Since mass loss due to winds is significantly reduced in metal-poor stars, the growth of a VMS should be more efficient at low metallicity and the resulting $\mathrm{BH}$ mass can be as high as $10^{3} \mathrm{M}_{\odot}$ (Devecchi \& Volonteri 2009; Volonteri 2010).

Hence, in GQd we assume that a medium-weight seed forms in atomic cooling halos $\left(T_{\mathrm{vir}} \geq 10^{4} \mathrm{~K}\right)$ where the gas is illuminated by a LW flux with $J_{\mathrm{LW}} \geq J_{\mathrm{cr}}$, the metal content of the ISM is subcritical, $Z<Z_{\mathrm{cr}}$, but the dust content is supercritical, $\mathcal{D} \geq \mathcal{D}_{\text {cr }}$, so that dust cooling can lead to the formation of a dense star cluster. In addition, we require the halo to have a sufficiently large reservoir of gas, $M_{\text {gas }} \geq$ $10^{6} \mathrm{M}_{\odot}$, to form a dense and compact star cluster and we assume its final fate to be the formation of a medium-weight seed with a mass of $10^{3} \mathrm{M}_{\odot}$.

The environmental conditions required to form light, medium-weight, and heavy seeds in GQd are summarized in Table 2. In section 4, we discuss the effects of varying the adopted critical threshold for the LW flux to suppress $\mathrm{H}_{2}$ formation. In addition, following Chon \& Omukai 2020, we also consider a variant of the reference model where we account for super-competitive accretion, that we illustrate below.

\subsection{The super-competitive accretion model}

In a recent work Chon \& Omukai (2020) (see also Tagawa et al. 2020) used a suite of hydrodynamical simulations to investigate the outcome of star formation in slightly metal-enriched gas clouds illuminated by a very strong UV field. The initial conditions were set by extracting - from a cosmological simulation - one halo that was shown to lead to the formation of a heavy seed when the gas was metal-free (Chon et al. 2018). As expected from previous semi-analytical models (Omukai et al. 2008), dust-cooling becomes important when $Z \geq 5 \times 10^{-6} Z_{\odot}$ and multiple (about $600)$ low-mass fragments form in a relatively compact region with size smaller than 100 au. On larger scales, however, where dust cooling is not effective, the structure of the cloud is very similar to the primordial case: as a result of the high gas temperatures, matter continues to flow into the central regions, preferentially feeding the primary star (defined as the first protostar that forms). In $10^{4}$ yr the primary star becomes supermassive, with a mass comparable to the one found in the primordial case. Interestingly, a similar evolution is found for initial metallicities up to $Z=10^{-4} Z_{\odot}$, although the initial number of low-mass protostars formed as a result of dust-cooling increases with $Z$ (about 4000 stars are formed when $Z=10^{-4} Z_{\odot}$ ). When the metallicity becomes $Z=10^{-3} Z_{\odot}$ (the maximum metallicity considered in their analysis), Chon \& Omukai (2020) found that metal fine-structure line cooling starts to be effective on larger scales (when the central gas density is $n \geq 10^{4} \mathrm{~cm}^{-3}$ ), and the associated decrease in the gas temperature leads to a suppression of the gas accretion rate by almost two orders of magnitude. As a result, the primary star mass grows only up to $350 \mathrm{M}_{\odot}$. It is important to stress however, that some of the secondary protostars merge with the primary stars. Although the fraction of mass acquired through mergers is subdominant when $Z<10^{-4} Z_{\odot}$, mergers contribute to more than $50 \%$ of the final primary mass when $Z=10^{-3} Z_{\odot}$. Since the total stellar mass formed in this highest metallicity run is $\geq 10^{3} \mathrm{M}_{\odot}$, this may imply that the resulting primary star 
Table 2. The environmental conditions adopted to plant the three families of seeds in the GQd reference $(\mathrm{R})$ model and in the super-competitive accretion (SCA) variant (see sections 2.3 and 3 ). For each of these models, two values of $\boldsymbol{J}_{\mathrm{cr}}$ have been explored: 300 and 1000 .

\begin{tabular}{|c|c|c|c|c|}
\hline $\mathrm{R}$ model & $Z / Z_{\odot}$ & $\mathcal{D}$ & $J_{\mathrm{LW}}$ & $M_{\text {gas }}$ \\
\hline $\begin{array}{l}\text { light } \\
\text { medium } \\
\text { heavy }\end{array}$ & $\begin{array}{l}<10^{-3.8} \\
<10^{-3.8} \\
<10^{-3.8}\end{array}$ & $\begin{array}{l}<4.4 \cdot 10^{-9} \\
\geq 4.4 \cdot 10^{-9} \\
<4.4 \cdot 10^{-9}\end{array}$ & $\begin{array}{l}<J \mathrm{cr} \\
\geq J \mathrm{cr} \\
\geq J \mathrm{cr}\end{array}$ & $\begin{aligned} & >0 \\
> & 10^{6} \mathrm{M}_{\odot} \\
> & 10^{6} \mathrm{M}_{\odot}\end{aligned}$ \\
\hline SCA model & $Z / Z_{\odot}$ & $\mathcal{D}$ & $J_{\mathrm{LW}}$ & $M_{\text {gas }}$ \\
\hline $\begin{array}{l}\text { light } \\
\text { medium } \\
\text { heavy }\end{array}$ & $\begin{array}{c}<10^{-3.8} \\
{\left[10^{-3.5}-10^{-2.5}\right]} \\
\quad<10^{-3.5}\end{array}$ & $\begin{array}{c}<4.4 \cdot 10^{-9} \\
\text { all } \\
\text { all }\end{array}$ & $\begin{array}{l}<J \mathrm{cr} \\
\geq J \mathrm{cr} \\
\geq J \mathrm{cr}\end{array}$ & $\begin{aligned} & >0 \\
> & 10^{6} \mathrm{M}_{\odot} \\
> & 10^{6} \mathrm{M}_{\odot}\end{aligned}$ \\
\hline
\end{tabular}

may grow further if the evolution were followed for more than the $10^{4}$ yr allowed by the simulation.

Hence, in this super-competitive accretion (SCA) model, SMSs leading to heavy BH seeds may continue to form at higher metallicities than assumed in our reference model. To investigate how this alternative scenario would modify the relative importance of the different BH seed populations, we run additional simulations where the conditions to form medium-weight and heavy seeds are set as follows: atomic cooling halos illuminated by a super-critical LW flux host the formation of heavy seeds as long as $Z<10^{-3.5} Z_{\odot}$, and of medium-weight seeds when $10^{-3.5} Z_{\odot} \leq Z<10^{-2.5} Z_{\odot}$ independent of their dust-to-gas mass ratio. Conversely, the transition from Pop III to Pop II stars is still assumed to occur when $Z \geq Z_{\text {cr }}=10^{-3.8} Z_{\odot}$ and $\mathcal{D}>4.4 \times 10^{-9}$ so that this model variant does not directly affect the conditions to form light seeds (see Table 2).

\subsection{Inhomogeneous feedback}

In the GQd model, metals and dust returned to the ISM through SN explosions and AGB stellar winds are assumed to instantaneously mix in the ISM. In addition, SN- and AGN-driven outflows described in section 2 have a metal and dust composition that reflect the conditions at the outflow launching site. Once ejected in the IGM, we assume metals and dust to uniformly mix with the surrounding gas. This is the pre-enriched medium that will be accreted onto newly virialized dark matter halos, and out of which new stellar and/or BH seeds will form.

In Valiante et al. (2016b), the formation rate of light and heavy seeds was analyzed by applying the same conditions described in section 2.3 and summarized in Table 2 for the reference model. We found that, under the hypothesis of uniform metal/dust mixing and uniform LW background, the redshift window where halos satisfied the conditions for heavy seeds formation was extremely narrow, and only a few heavy seéds (between 3 and 30, depending on the simulation) were able to form. This number, however, was enough to trigger the Eddington-limited growth to a SMBH by $z \sim 6$ (Valiante et al. 2016b).

However, metal (and dust) enrichment is known to be a highly inhomogeneous process, particularly at high redshift. Cosmological hydrodynamic simulations (Tornatore et al. 2007; Maio et al. 2011; Wise et al. 2012;
Johnson et al. 2013; Muratov et al. 2013; Pallottini et al. 2015; Xu et al. 2016; Jaacks et al. 2018; Sarmento et al. 2019; Graziani et al. 2019) supported by observations (Robert et al. 2019; Vanzella et al. 2020) show that metals ejected in the ISM by SNe and AGB stars propagate from higher density peaks to lower density regions, allowing the co-existence of pristine and metal enriched objects in the pre-reionization epoch.

Similarly, although the intensity of the LW background produced by stellar sources and accreting black holes increases very rapidly in the biased region where the bright quasar and its host galaxy are assembling (see Fig. 5 of Valiante et al. 2016b), local fluctuations of the LW intensity from nearby sources can significantly exceed the background level, suppressing $\mathrm{H}_{2}$ cooling and aiding to win the competition between LW radiation and metal winds in heavy seeds hosts (Ahn et al. 2009; Regan et al. 2014; Dijkstra et al. 2014; Inayoshi \& Tanaka 2015; Habouzit et al. 2016a; Agarwal et al.2017; Maío et al. 2019; Mayer \& Bonoli 2019).

Since we do not have any information on the spatial distribution of dark matter halos in the simulated volume, or on the spatial distribution of metal-enriched bubbles, we have implemented in GQd a statistical description of inhomogeneous chemical and radiative feedback that we present below.

\subsubsection{Inhomogeneous metal and dust enrichment}

Following Dijkstra et al. (2014) and Salvadori et al. (2014), at each redshift we compute the volume filling factor of the $\mathrm{N}$ enriched regions as:

$Q(z)=\sum_{\mathrm{i}=1}^{\mathrm{N}} \frac{V_{\mathrm{i}}}{V_{\text {sim }}}=\sum_{\mathrm{i}=1}^{\mathrm{N}} \frac{4 / 3 \pi R_{\mathrm{i}}^{3}}{V_{\text {sim }}}$,

where $\sum_{\mathrm{i}=1}^{\mathrm{N}} V_{\mathrm{i}}$ is the volume filled by $\mathrm{SN}$-driven metalenriched bubbles with radius $R_{i}$ and $V_{\text {sim }}=50 \mathrm{Mpc}^{3}(1+z)^{-3}$ is the proper volume of the simulation, estimated at the turn-around radius. At a given time (redshift), the radius of the $i$-th expanding bubble polluted by metals can be approximated by the self-similar Sedov-Taylor blastwave solution (Madau et al. 2001; Dijkstra et al. 2014):

$R_{\mathrm{i}}(t)=\xi_{0}\left[\frac{E_{\mathrm{SN}} N_{\mathrm{SN}, \mathrm{i}}}{\rho_{\mathrm{gaS}}\left(z_{\mathrm{i}}\right)}\right]^{1 / 5} t^{2 / 5}$,

where $\xi_{0}=1.17, E_{\mathrm{SN}} N_{\mathrm{SN}, \mathrm{i}}$ is the total SN explosion energy in the i-th halo $\left(E_{\mathrm{SN}}\right.$ is the averaged energy per $\mathrm{SN}$, as in Eq. 2.7 , and $N_{\mathrm{SN}}$ is the number of $\left.\mathrm{SNe}\right)^{3} \rho_{\text {gas }}$ is the gas density and $t=t(z)-t\left(z_{\mathrm{i}}\right)$ is the interval between the current time $z$ and the redshift $z_{\mathrm{i}}$ at which the SN-driven outflow was launched. When the bubble is within the halo virial radius, $R_{\mathrm{i}}<R_{\mathrm{vir}, \mathrm{i}}, \rho_{\text {gas }}$ is the mean gas density of the $\mathrm{i}$-th halo at the moment of the $\mathrm{SNe}$ explosion. As the bubble expands

3 During the Sedov-Taylor expansion phase the energy is conserved and not radiated away. Hence, in Eq.3.2, we consider all the energy (kinetic and thermal) injected by each SN. 
beyond the halo virial radius into the IGM, $R_{\mathrm{i}}>R_{\mathrm{vir}, \mathrm{i}}$, we compute the gas density ${ }^{4}$ as $\rho_{\text {gas }}(z)=\Omega_{\mathrm{b}} \rho_{\mathrm{cr}}(1+z)^{3}$.

At a given time (redshift) we define the mean metallicity, $Z_{\mathrm{q}}$, and dust-to-gas mass ratio, $\mathcal{D}_{\mathrm{q}}$ of the enriched regions as:

$Z_{\mathrm{q}}(t)=\frac{M_{\mathrm{met}, \mathrm{ej}}^{\mathrm{tot}}(t)}{M_{\mathrm{ej}}^{\mathrm{tot}}(t)+\left[1-f_{\mathrm{c}}(t)\right] \sum_{\mathrm{i}=1}^{\mathrm{N}} \Omega_{\mathrm{b}} \rho_{\mathrm{cr}}(1+z)^{3} V_{i}}$,

and

$$
\mathcal{D}_{\mathrm{q}}(t)=\frac{M_{\mathrm{dust}, \mathrm{ej}}^{\mathrm{tot}}(t)}{M_{\mathrm{ej}}^{\mathrm{tot}}(t)+\left[1-f_{\mathrm{c}}(t)\right] \sum_{\mathrm{i}=1}^{\mathrm{N}} \Omega_{\mathrm{b}} \rho_{\mathrm{cr}}(1+z)^{3} V_{i}} .
$$

where $M_{\mathrm{ej}}^{\mathrm{tot}}(t), M_{\mathrm{met}, \mathrm{ej}}^{\mathrm{tot}}(t)$ and $M_{\mathrm{dust}, \mathrm{ej}}^{\mathrm{tot}}(t)$ are the total masses of gas, metals and dust that have been ejected out of the halos in the IGM, $\left[1-f_{\mathrm{c}}(t)\right] \sum_{\mathrm{i}=1}^{\mathrm{N}} \Omega_{\mathrm{b}} \rho_{\mathrm{cr}}(1+z)^{3} V_{i}$ is the IGM gas mass enclosed in the volume covered by the bubbles expanding outside the halos (the summation runs on all halos for which the condition $R_{\mathrm{i}}>R_{\mathrm{vir}, \mathrm{i}}$ is satisfied), and $f_{\mathrm{c}}(t)$ is the time-dependent fraction of mass collapsed into halos. The amount of gas ejected out of a single halo as a consequence of mechanical feedback, $M_{\mathrm{ej}}$, is computed as described in section 2. The corresponding mass of ejected metals and dust are computed as $M_{\text {met,ej }}=Z_{\mathrm{ISM}} M_{\mathrm{ej}}$ and $M_{\text {dust,ej }}=\mathcal{D}_{\mathrm{ISM}} M_{\mathrm{ej}}$, where $Z_{\text {ISM }}$ and $\mathcal{D}_{\text {ISM }}$ are the metallicity and dust-to-gas ratio of the ISM (Valiante et al. 2011, 2014).

At each redshift, we assume that the $i$-th halo has a probability $P_{\mathrm{i}}(z) \in[0,1]$, randomly extracted from a uniform distribution, to be located within a region of the IGM that has been (already) enriched by SN explosions (i.e. regions occupied by expanding bubbles). We then use the volume filling factor, $Q(z)$, to assign halos to the un-polluted or to the enriched volume: if $P_{\mathrm{i}}(z)>Q(z)$, the halo resides in the un-polluted volume $[1-Q(z)] V_{\text {sim }}$, where it accretes gas of primordial composition; if $P_{\mathrm{i}}(z) \leq Q(z)$, the halo is instead associated to enriched regions, within the volume $Q(z) V_{\text {sim }}$, and accretes polluted gas from the $\mathrm{IGM}^{5}$. At each given time, $Z_{\mathrm{q}}$ and $\mathcal{D}_{\mathrm{q}}$ define the abundance of metals and dust in the (infalling) material accreted by halos forming/evolving in enriched regions, as described above. Figure 2 shows the redshift evolution of the filling factor ( $Q$, left-hand panel), metallicity $\left(Z_{\mathrm{q}}\right.$, central panel), and dust-to-gas mass ratio $\left(\mathcal{D}_{\mathrm{q}}\right.$, right-hand panel) of enriched regions predicted by the reference model (see section 4 ). While $Q$ monotonically increases with cosmic time, reaching the value $Q=1$ at $z \sim 16$, the redshift evolution of $Z_{\mathrm{q}}$ and $\mathcal{D}_{\mathrm{q}}$ is not monotonic and depends on the metal and dust yields of the dominant SN population, on their energies, and on the mass of gas swept by the expanding bubbles. At early times, when the SFR is dominated by Pop AII stars, the bubbles are still relatively small $(Q<<1)$ and there is a very rapid increase in both $Z_{\mathrm{q}}$ and $\mathcal{D}_{\mathrm{q}}$, implying that the few enriched regions are

4 We note that this may lead to an unphysical jump in the gas density as the bubble reaches the virial radius. However, adopting a smoother transition, such as the one predicted by Madau et al. (2001), delays the time evolution of the shell radius for a very small time interval and provides a negligible correction to the time evolution of the filling factor.

5 Note that, in the limit $Q(z) \rightarrow 1$, the IGM is completely filled by metal-enriched bubbles and the enriched volume converges to the total volume of the simulation: $\sum_{\mathrm{i}=1}^{\mathrm{N}} V_{\mathrm{i}} \rightarrow V_{\text {sim }}$.

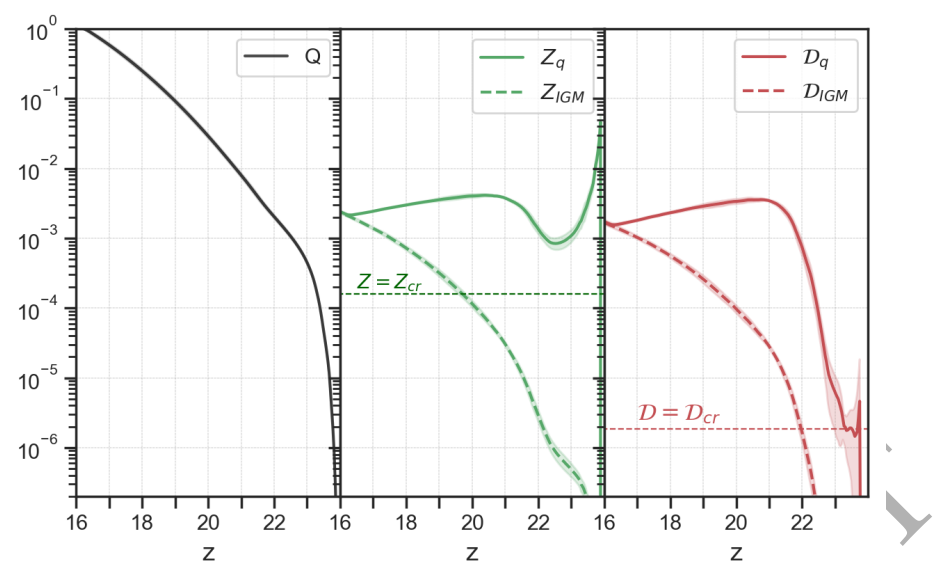

Figure 2. Left panel: evolution of the filling factor of enriched regions (black line) as a function of redshift. Central panel: mean metallicity of the IGM (green dashed line) and of the enriched regions (green solid line) expressed in solar units, with $Z_{\odot}=0.02$. Right panel: mean dust-to-gas ratio of the IGM (red dashed line) and of the enriched regions (red solid line) normalized to the Galactic value, $\mathcal{D}_{\mathrm{MW}}=0.006$. The lines are mean values over ten realizations of the reference model, with 1- $\sigma$ errorbars (light shaded areas). Horizontal dashed lines in the central and right panels are the critical values for metallicity $\left(Z_{\mathrm{cr}}\right)$ and dust to gas ratio $\left(\mathcal{D}_{\text {cr }}\right)$ adopted in the reference model.

very metal- and dust-rich. As the bubbles expand, progressively enclosing a larger IGM gas mass, $Z_{\mathrm{q}}$ and $\mathcal{D}_{\mathrm{q}}$ decrease. When the star formation rate starts to be dominated by Pop II stars, at $z \lesssim 23, Z_{\mathrm{q}}$ and $\mathcal{D}_{\mathrm{q}}$ increase again but then smoothly decline until, at $z \sim 16$, when $Q \sim 1$, the volume is homogeneously enriched and their values converge to the mean IGM metallicity, $Z_{\mathrm{IGM}}$, and dust-to-gas mass ratio, $\mathcal{D}_{\text {IGM }}$ (shown in Fig. 2 as dashed lines). The two horizontal lines in Fig. 2 represent the adopted critical metallicity and dust-to-gas ratio in the reference model (see Table 2). The figure shows that the condition $Z_{\mathrm{IGM}} \sim Z_{\mathrm{cr}}\left(\mathcal{D}_{\mathrm{IGM}} \sim \mathcal{D}_{\mathrm{cr}}\right)$ is met already at $z \sim 20(z \sim 22)$. These are the reshifts below which $\mathrm{BH}$ seeds can no longer form in models where the IGM is assumed to be uniformly enriched. However, at these cosmic epochs, we predict the filling factor to be still very small $\left(Q \sim 10^{-2}\right)$ and metal enrichment to be highly inhomogeneous. As a consequence, $\mathrm{BH}$ seeds can continue to form down to $z \sim 16$, when the volume has been completely filled by metal-enriched bubbles $(Q=1)$. Hence, inhomogeneous enrichment extends the redshift range where BH seeds may potentially form from $z \sim 20$ to $z \sim 16$.

As a final remark, we notice that the above model of inhomogeneous enrichment assumes that the individual enriched bubbles do not overlap. This neglects the effects of clustering, which are likely to be significant for high-redshift star-forming galaxies and may lead to a $\sim 70 \%$ reduction of $Q$ at $z<17$, assuming that the enrichment is mostly driven by $10^{6}-10^{7} M_{\odot}$ halos $^{6}$. The effects of this reduction would be to

6 The average comoving distance between $10^{6}-10^{7} M_{\odot}$ halos at $z=10-20$ ranges between $\langle d\rangle \sim 0.13$ to $\sim 1.1 \mathrm{Mpc}$. The excess 
extend the epoch of $\mathrm{BH}$ seed formation to lower redshift and to favour the formation of heavy $\mathrm{BH}$ seeds, that preferentially form in metal-free atomic-cooling halos illuminated by a super-critical LW flux, at the expenses of medium-weight $\mathrm{BH}$ seeds (see section 2.3). However, this does not significantly affect the resulting evolution of the SMBH mass (see section 2.4).

\subsubsection{Inhomogeneous $J_{L W}$ flux}

Inhomogeneities in the LW flux and their relation with potential sites for heavy BH seeds formation have been investigated for many years (Dijkstra et al. 2008; Ahn et al. 2009; Agarwal et al. 2012; Dijkstra et al. 2014; Inayoshi \& Tanaka 2015). In fact, in the redshift range where BH seeds are expected to form, the mean LW background is significantly smaller than the required critical value above which $\mathrm{H}_{2}$ cooling in atomic cooling halos is suppressed, unless the region under investigation is particularly over-dense (Valiante et al. 2016a,b). Hence, heavy seeds form when their potential host halo is illuminated by a super-critical $J_{\text {LW }}$ from a nearby galaxy (Visbal et al. 2014; Habouzit et al. 2016b; Regan et al. 2019; Maio et al. 2019), whose distance must not be too close in order to avoid gas stripping or tidal distortions, which would prevent the formation of the SMS (Chon et al. 2016).

This motivates the importance of considering the nonlinear spatial distribution of the UV emitting sources around a certain atomic cooling halo, $M_{1}$, candidate to host a heavy or medium-weight seed. In other words, the proximity of $M_{1}$ to a star-forming halo can easily help the halo to meet the condition that $J_{\mathrm{LW}} \geq J_{\mathrm{cr}}$.

To establish the distance between $M_{1}$ and a UV source, we start by considering the probability to find another halo at distance $r$. Following Inayoshi \& Tanaka (2015), the differential probability distribution of finding a halo with mass $M_{2} \pm d M / 2$ at a distance $r \pm d r$ from $M_{1}$ is given by

$\frac{d^{2} P\left(M_{1}, M_{2}, z, r\right)}{d M d r}=4 \pi r^{2}(1+z)^{3} \times\left[1+\xi\left(M_{1}, M_{2}, z, r\right)\right] \frac{d n}{d M},(3.5)$ where $d n / d M$ is the comoving number density of $M_{2}$ halos at redshift $z$ and $\xi$ is the non-linear bias (Hliev et al. 2003).

At each redshift of the merger tree, we identify - among all simulated halos - those candidate to host a BH seed (see section 2.3 and Table 2), $M_{1}$. For each of these, we consider $N_{r}$ spherical shells centered on $M_{1}$, with radii $r_{i}$ varying from $R_{\text {min }}=10^{-3} \mathrm{Mpc}$ to $R_{\max }=\left(3 V_{\mathrm{sim}} / 4 \pi\right)^{1 / 3} \mathrm{Mpc}$. We then randomly sample the cumulative probability to find, among all possible star-forming halos $N_{s}$, a source $j$ at distance $r_{i}$ and we compute the LW intensity that it emits due to star formation, $I_{\text {stars }}^{j}$ and $\mathrm{BH}$ accretion $I_{\mathrm{AGN}}^{j}$. Finally, we sum all the contributions to compute the total LW flux irradiating

probability of finding two halos with mass $M=10^{6} M_{\odot}\left(10^{7} M_{\odot}\right)$ separated by a comoving distance $<d>$ at $10<z<20$ can be estimated to range between 1 and 3 (see e.g. Fig. 2 in Iliev et al. 2003). Assuming that within $\langle d\rangle$ we find 3 times more systems than if these were uniformly distributed, and that their individual metal-enriched bubbles overlap, our estimated filling factor should be reduced by $\sim 70 \%$ to account for this clustering effect. each $M_{1}$ :

$J_{\mathrm{LW}}=\sum_{j=1}^{N_{s}} \sum_{i=1}^{N_{r}} \frac{\left(I_{\mathrm{stars}, \mathrm{i}}^{j}+I_{\mathrm{AGN}, \mathrm{i}}^{j}\right)}{4 \pi r_{i}^{2}}$.

For the present study, we follow Iliev et al. (2003) and adopt $N_{r}=300 . I_{\text {stars }}^{j}$ is computed using the mass (or age) and metallicity-dependent emissivities by Schaerer (2002) (for Pop III stars) and Bruzual \& Charlot (2003) (for Pop II/I stars) while $I_{\mathrm{AGN}}^{j}$ is obtained from a standard multitemperature disc model with the addition of a non-thermal power-law component at high energies ( $\propto v^{-2}$ Shakura 1973; Sazonov et al. 2004).

\section{RESULTS}

In this section, we start by illustrating the results of the reference model, R300, where we adopt a critical value for the LW flux of $J_{\mathrm{cr}}=300$. We first present the properties of $\mathrm{BH}$ seeds formation environments, such as the intensity of the illuminating LW radiation, $J_{\mathrm{LW}}$, the metallicity, and dust-to-gas mass ratio. Then, we quantify the relative contribution of different seed populations to the mass growth history of the final SMBH. We finally explore the implications of changing the value of $J_{\text {cr }}$ and how the growth history of the SMBH is modified when the SCA model is adopted.

\subsection{Mean and local LW flux}

In Fig. 3, we show the predicted evolution of the mean LW background as a function of redshift (blue lines) in 10 independent simulations of R300. It is important to stress that this quantifies the LW background in our simulated volume that represents a very biased region that will form a massive halo at $z=6.4$. This is why the solid lines reach values that are larger than the cosmic averages typically found in other studies (see, for instance, Fig. 6 in Ahn et al. 2009 or Fig. 3 in Dijkstra et al. 2014 where their mean LW background at $z>10$ is found to be in the range $\sim 0.1-10$ ). Since the mean LW background is roughly proportional to the stellar mass density (see Eqs. 12 and 13 in Agarwal et al. 2012), a larger background is to be expected in the highly biased region that will assemble a $10^{13} M_{\odot}$ halo at $z=6.4$. Indeed, Petri et al. (2012) find comparably large values when they simulate the assembly of a $10^{12} M_{\odot}$ halo at $z \sim 6$ (see their Fig. 5).

Conversely, our predicted local fluctuations in the LW field are very consistent with previous findings (see, for example, Fig. 3 of Agarwal et al. 2012 or the colour map in Fig. 1 of Dijkstra et al. 2014). These are shown with grey points in Figure 3, that represent the (local) intensity of LW radiation illuminating individual halos. Among these, we have marked in red (yellow) the formation sites of heavy (medium-weight) seeds. Red dotted lines identify the redshift at which the mean LW background exceeds the critical value $J_{\text {cr }}=300$. In all simulations, this condition is met at $z \sim 21$. While medium-weight seeds can form above this redshift, we find that there are large fluctuations around the mean LW background and in most cases heavy and mediumweight seeds form in environments where $J_{\mathrm{LW}}>10^{3}$, largely exceeding our adopted value for $J_{\text {cr. }}$. 


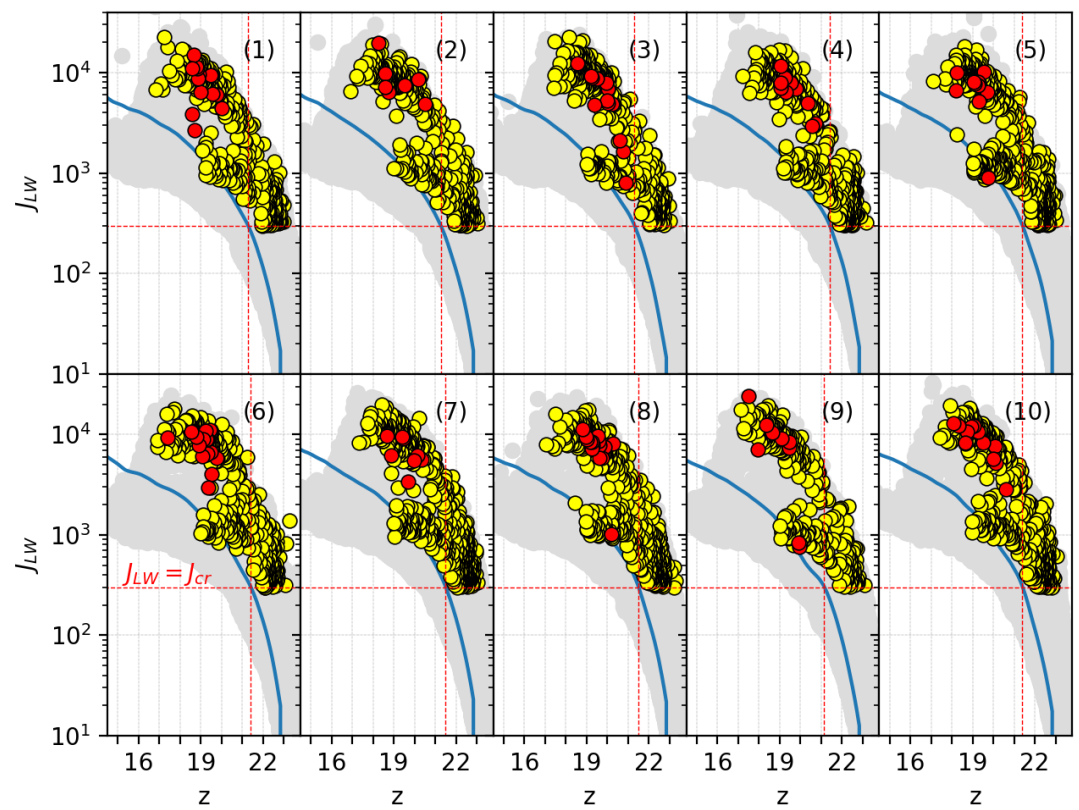

Figure 4. The ISM dust-to-gas mass ratio and metallicity of all the star-forming galaxies in simulation (10) at $z \geq 16$. Each grey point represents a single halo and - similarly to Fig. 3 - we have marked in red and kellow the formation sites of heavy and medium-weight $\mathrm{BH}$ seeds. We note that the majority of heavy seeds form in pristine galaxies that are not accounted for in this plane. Cyan and blue points indicate, respectively, non-pristine minihalos and atomic-cooling halos where light seeds form. The black solid line represents the mean values of the IGM. Red dashed lines are the critical dust-to-gas ratio and metallicity threshelds adopted in the reference model. For comparison, the two thin vertical lines show the critical metallicity thresholds adopted in the SCA model (see Fig. 8 and Table 2)

\subsection{Mean and local metal enrichment}

In Fig. 4, we report the ISM metallicity and dust-to-gas mass ratios of individual galaxies extracted from a single simulation (simulation 10 shown in the bottom-right panel of Fig. 3) from the onset of chemical enrichment down to the redshift at which the filling factor of enriched regions, $Q$, is equal to $1(z \geq 16)$. We follow the same colour code adopted in Fig. 3 and we mark in red and yellow systems hosting heavy and medium-weight $\mathrm{BH}$ seeds, respectively. In addition, we also show the formation sites of light BH seeds, marking in cyan and blue systems hosted, respectively, in minihalos and atomic cooling halos. The solid black line indicates the mean values of the IGM, that shows a monotonic increase of $\mathcal{D}_{\text {IGM }}$ with $Z_{\text {IGM }}{ }^{7}$. As expected from Fig. 2, when $Q<<1$, galaxies located within the already enriched regions have a metallicity and dust-to-gas mass ratio that largely exceed the mean IGM value (and above the critical metallicity and dust-to-gas mass ratio thresholds) populating the upper-right end of Fig. 4, together with their lower-redshift and metal/dust-rich descendants. On the other hand, unpolluted regions at $z>16$ host metal/dust-free $(Z=\mathcal{D}=0)$ galaxies that do not appear in the figure. This is why the density of grey points is significantly smaller in the bottomleft part of the plane.

The two red dashed lines mark the critical values for $Z_{\mathrm{cr}}$ and $\mathcal{D}_{\mathrm{cr}}$ adopted in the reference model. These divide the plane in four separate regions: the bottom-left part contains the most pristine environments, where only light and heavy $\mathrm{BH}$ seeds form. We note that the number of red points is sig-

7 We point out that $\mathcal{D}$ and $Z$ are not always proportional because of dust reprocessing in the ISM due to SN dust destruction and grain growth (see section 3.3 ). 

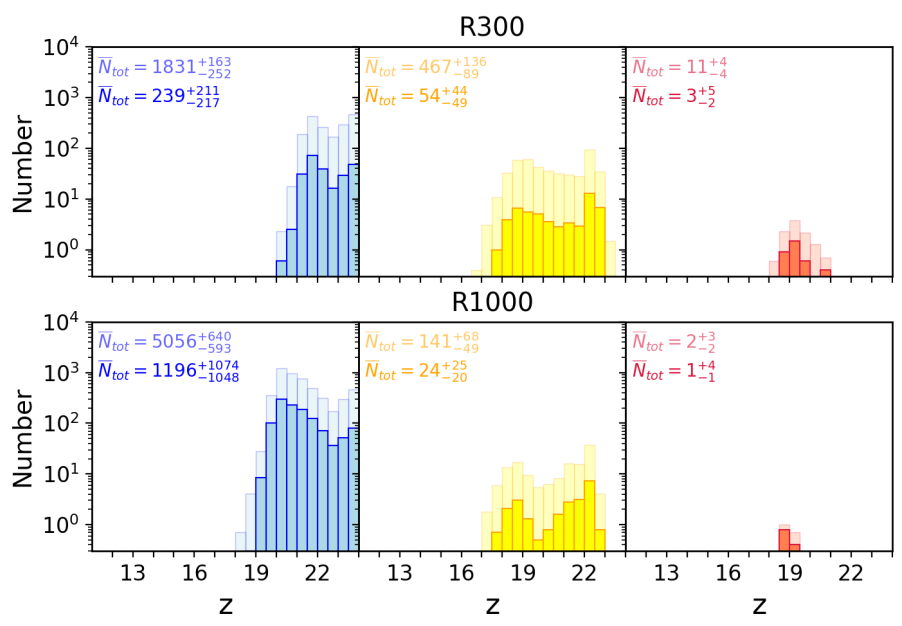

Figure 5. Distribution of formation redshifts of light, mediumweight, and heavy BH seeds (from left to right). The histograms indicate the mean values averaged over 10 different simulations. Histograms in lighter colours show the global BH seed population, whereas histograms in heavier colours illustrate the true progenitors of the final SMBH at $z=6.4$ (see text). In each panel, we also indicate the total number of seeds formed, on average, in the corresponding population. The errors indicate the difference with the maximum and minimum values for each family over 10 simulations. We compare the results for the same reference set of parameters, assuming $J_{\mathrm{cr}}=300$ (model R300, top panels) and $J_{\mathrm{cr}}=1000$ (model R1000, bottom panels).

nificantly smaller than Fig. 3 (see the bottom-right panel). In fact, most of the heavy $\mathrm{BH}$ seeds form in pristine halos, that are not visible in Fig. 4.

A similar consideration applies to light $\mathrm{BH}$ seeds, a large fraction of which form when $Z=\mathcal{D}=0$. In the top-left region only medium-weight seeds can form, in the sub-sample of halos that are illuminated by a super-critical $J_{\text {LW }}$ and that contain a sufficiently large gas mass $\left(\geq 10^{6} \mathrm{M}_{\odot}\right)$. The latter condition preferentially selects systems along diagonal lines on this plane. The highest density of galaxies appears to the right of the vertical line representing $Z=Z_{\text {cr }}$, where Pop II/I stars are assumed to form by metal and dust-driven fragmentation. Indeed, their parent star-forming clouds are either initially enriched above $\mathcal{D}_{\mathrm{cr}}$ (top-right region) or they reach this condition during cloud collapse through grain growth (Chiaki et al. 2014, 2015).

\subsection{Seeds birth environments}

In the top panel of Figure 5, we show the distribution of formation redshifts of light, medium-weight, and heavy $\mathrm{BH}$ seeds for model R300. The bottom panel will be discussed in section 4.5. The light-coloured histograms represent the averaged values found in 10 independent simulations and in each panel we also report the corresponding total number of BH seeds formed. Histograms in heavier colours illustrate the sub-sample of each family of seeds that are the progenitors of the final SMBH at $z=6.4$ (those systems that directly contribute to its mass assembly $\left.{ }^{8}\right)$. The errors associated to the number of $\mathrm{BH}$ seeds (and $\mathrm{BH}$ seeds progenitors) correspond to the difference with the maximum and minimum values found in the 10 simulations.

We find that the epoch of formation of $\mathrm{BH}$ seeds starts at $z \sim 24$, when $\mathrm{H}_{2}$ cooling in the first mini-halos becomes efficient enough to trigger star formation ${ }^{9}$. As expected, light $\mathrm{BH}$ seeds form first and largely outnumber medium-weight and heavy BH seeds. On average, we find $\sim 1831$ light $\mathrm{BH}$ seeds forming at $z \geq 20$, while $\sim 467$ medium-weight and $\sim 11$ heavy $\mathrm{BH}$ seeds form at $16 \leq z \leq 23.5$, when atomic-cooling halos start to assemble. Termination of light $\mathrm{BH}$ seed formation is set by radiative feedback, as at $z \leq 20$ metal-poor or pristine star-forming regions are illuminated by a supercritical LW flux (see Fig. 3) and meet the condition to form medium-weight and heavy BH seeds. We note that mediumweight seeds start to form before their heavy counterparts. Indeed, while the latter preferentially form in pristine halos, the former can seed halos that have already experienced previous episodes of star formation but whose $Z$ and $\mathcal{D}$ still meet the conditions required to form medium-weight seeds.

The above results can be compared to Valiante et al. (2016b, see in particular their Fig. 4). While the results of the above study are qualitatively similar, our inhomogeneous treatment of chemical and radiative feedback leads to an increase in the absolute number of light seeds $(\sim 830$ light and $\sim 10$ heavy BH seeds were predicted to form, on average, in Valiante et al. 2016b) and to a more uniform distribution in the formation epoch of heavy BH seeds (the majority of which where expected to form at $z \sim 16$ in Valiante et al. $2016 \mathrm{~b})$, thanks to the higher number of pristine regions and to the fluctuations in the LW flux.

The comparison between lighter and darker histograms in each panel shows that the formation redshift distributions of $\mathrm{BH}$ seeds progenitors follow the same trend of their parent populations but with smaller numbers: on average, only $12-13$ per cent of medium-weight and light and $\sim 27$ per cent of heavy BH seeds are progenitors of the final SMBH.

As a final remark, we stress that these absolute numbers quantify the seeds populations within a comoving volume of $50 \mathrm{Mpc}^{3}$ selected to host a $10^{13} \mathrm{M}_{\odot}$ dark matter halo at $z=6.4$. Hence, it is not straightforward to interpret these numbers as representative of an average cosmic region of the Universe. Rather, they should be considered as the expected $\mathrm{BH}$ population along the formation route of each $z>6 \mathrm{SMBH}$

8 As explained in section 2.4, the progenitors of the final SMBH are seed BHs that - while growing - remain in the nuclei of their galaxies and never become wandering $\mathrm{BHs}$ as a consequence of minor mergers experienced by their host halo.

9 According to Valiante et al. (2016b), the fraction of metal-free gas that is able to cool in one dynamical time, $f_{\text {cool }}$ (see Eq. 1), rapidly drops to zero when $T_{\mathrm{vir}}<2000 \mathrm{~K}$ at $z \geq 25$, even when $J_{\mathrm{LW}}=0$ (see the top left panel in Fig. A1 of Valiante et al. 2016b). This limit corresponds to a minimum halo mass that can host star formation of $\sim 10^{6} \mathrm{M}_{\odot}$ at $z \sim 24$, consistent with our resolution mass in the merger tree simulations. 


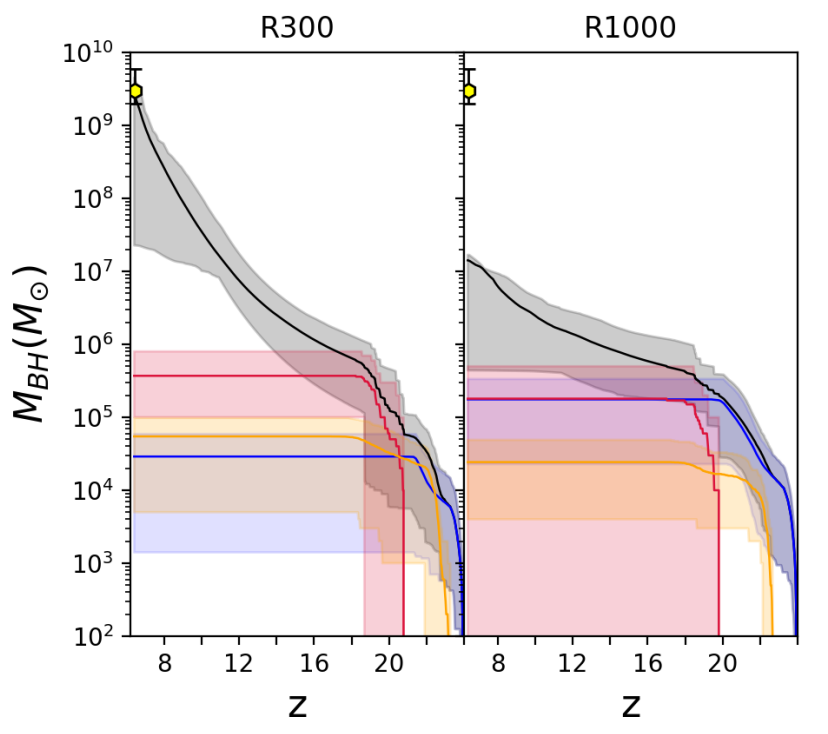

Figure 6. Evolution of the total mass of nuclear black holes as a function of redshift (black line) with the separate contributions of light (blue line), medium-weight (yellow line), and heavy BH seed (red line) progenitors. Each line represents the average value among 10 simulations, with the shaded region ranging between the minimum and maximum value found at each redshift. We compare the results for the same reference set of parameters but assuming $J_{\mathrm{cr}}=300$ (model R300, left panel) and $J_{\mathrm{cr}}=1000$ (model R1000, right panel). The data point at $z=6.4$ represents the estimated SMBH mass of the QSO $J 1148$ that we have assumed as a prototypical system and against which we have calibrated the model free parameters.

\subsection{Nuclear BH mass evolution}

In the left panel of Fig. 6, we show the resulting evolution of the nuclear BH mass as a function of redshift for model R300. This is obtained by summing over all BH seed progenitors masses ${ }^{10}$ present at each redshift in the nuclei of the simulated galaxies, starting from the multiplicity of small systems hosted in minihalos at $z=24$, down to the single $10^{13} \mathrm{M}_{\odot}$ halo at $z=6.4$. The figure shows the average evolution found among 10 different simulations considering accretion (black line) and illustrates the separate contributions to the total mass growth of light (blue line), mediumweight (yellow line), and heavy (red line) BH seed progenitors, without taking into account their subsequent growth by gas accretion. For each of these lines, the shaded area with the same colour ranges between the minimum and maximum values found at each redshift.

As expected from Fig. 5, the sequence of coloured lines reflects the formation redshifts of the three $\mathrm{BH}$ seeds populations. Despite their smallest number, the greatest contribution to the mass growth is provided by heavy seeds, (with

10 It is important to stress that we restrict the analysis to real SMBH progenitors, meaning that we reconstruct the mass and redshift distributions of all the nuclear $\mathrm{BHs}$ that contribute to the final SMBH mass. a cumulative $\mathrm{BH}$ mass of $\left.\sim 3 \times 10^{5} \mathrm{M}_{\odot}\right)$, that is much larger than those of light $\left(\sim 3 \times 10^{4} \mathrm{M}_{\odot}\right)$ and medium-weight seeds $\left(\sim 5 \times 10^{4} \mathrm{M}_{\odot}\right)$. At $z<16$, BH seeds no longer form and the growth of nuclear BHs is entirely driven by gas accretion, which provides the dominant contribution to the final SMBH mass of $\sim 3 \times 10^{9} \mathrm{M}_{\odot}$.

The above findings appear to be in very good qualitative agreement with the results obtained by Valiante et al. (2016b, see in particular their Fig. 3), where they also find that the Eddington-limited growth of $z \sim 6 \mathrm{SMBHs}$ relies on the formation of a small number of heavy seeds (with a total $\mathrm{BH}$ mass of $\sim 10^{6} \mathrm{M}_{\odot}$ ) at early times and is dominated by gas accretion at late times. While the present study confirms this qualitative picture, it also shows that medium-weight $\mathrm{BH}$ seeds appear to be more frequent than heavy seeds among the ancestors of $z \sim 6$ SMBHs but due to their smaller BH masses - their contribution to the total mass growth is largely subdominant.

\subsection{Dependence on the critical LW flux}

The results presented above haye been obtained selecting the environmental conditions to plant the three families of $\mathrm{BH}$ seeds according to a set of parameters that characterizes the reference model (see Table 2). Here we wish to explore the sensitivity of our results to the adopted critical value of $J_{\mathrm{cr}}$. Indeed, the critical value of the LW flux for heavy (and medium weight) $\mathrm{BH}$ seed formation is admittedly very uncertain. The recent review by Inayoshi et al. (2019) provides a thorough discussion of the various results that have been found by different groups using one-zone models or full $3 \mathrm{D}$ simulations. In general, the value of $J_{\text {cr }}$ depends on the details of the calculation of the optically-thick $\mathrm{H}_{2}$ photo-dissociation rate, which is challenging even in onezone models. Using emissivity properties that are realistic for low-metallicity galaxies, Sugimura et al. (2014) have provided the most complete calculations, finding $J_{\text {cr }}$ to vary in the range $\sim 1000-1400$, with the lower end that is generally favoured when self-shielding and non-LTE effects are considered (Wolcott-Green et al. 2017; Wolcott-Green et al. 2020). Full 3D simulations, that account for dynamical effects but generally implement simplified treatments of the shielding factor, tend to find values of $J_{\mathrm{cr}}$ that are a factor of few higher than in one-zone models (see Inayoshi et al. 2019 and references therein).

To explore the impact of this parameter on our results, we have run a set of new simulations of the reference model adopting the increased value of $J_{\mathrm{cr}}=1000$ (model R1000), as suggested by Sugimura et al. (2014). A comparison between model R1000 and the reference simulation (model R300) is provided in Figs. 5, 6, and 7 .

A major difference between the two models is the decrease in the number of medium-weight and heavy BH seeds in model R1000 (by a factor of $\sim 3.3-5.5$ ), partly compensated by a comparable increase of light BH seeds (see Fig. 5). Indeed, a larger $J_{\text {cr }}$ implies a less effective radiative feedback and that star formation in metal-free or metal-poor systems, that rely on $\mathrm{H}_{2}$ cooling, is no longer suppressed (i.e. all systems exposed to $300<J_{\mathrm{LW}}<1000$ can form stars in $\mathrm{R} 1000)$. Yet, due to the fluctuations of the LW radiation, halos that host the formation of a medium-weight or heavy $\mathrm{BH}$ seeds can be illuminated by a LW flux that largely exceeds 


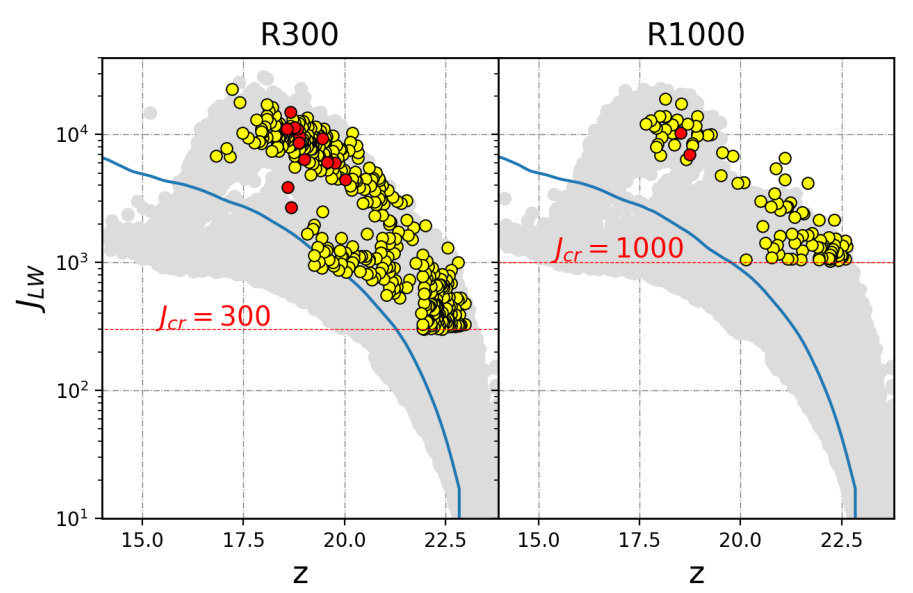

Figure 7. Same as Fig. 3 but for a single simulation and comparing the results of the reference model with $J_{\mathrm{cr}}=300$ (model R300, left panel, i.e. run 1 of Fig. 3 ) and $J_{\mathrm{cr}}=1000$ (model R1000, right panel).

$J_{\text {cr. }}$. This is clearly shown in Fig. 7 , that is the analogous of Fig. 3, but comparing the results of a single simulation in models R300 (left panel) and R1000 (right panel). In model R300 a large fraction of medium-weight seeds and all heavy $\mathrm{BH}$ seeds are predicted to form in systems illuminated by a LW intensity $>2000$. Hence, their formation should only be mildly affected by an increase in $J_{\text {cr }}$ from 300 to 1000 . However, the strong interplay between radiative and chemical feedback at these redshifts is such that the less effective radiative feedback leads to an increase in the (Pop III) SFR and to a more efficient metal and dust enrichment at these epochs. Due to star formation in their progenitors, most of the halos that host the formation of a medium-weight or heavy BH seed in model R300 are already too enriched in model R1000 and no longer meet the conditions to form a $\mathrm{BH}$ seed.

Similar considerations apply if we restrict the analysis to the population of $\mathrm{BH}$ seed progenitors, represented by the darker coloured histograms in the bottom panels of Fig. 5 . The increased value of $J_{\text {cr }}$ in modelR1000 leads to an increase by a factor $\sim 5$ in the number of light $\mathrm{BH}$ seed progenitors and to a factor $\sim 2.2-3$ reduction in the number of medium-weight and heavy BH seed progenitors with respect to model R300. As a result, Figure 6 shows that the Eddington-limited growth of the $\mathrm{BH}$ seed progenitors formed in model R1000 leads to the formation of a $z \sim 6 \mathrm{SMBH}$ with an average mass of only $\sim 2 \times 10^{7} \mathrm{M}_{\odot}$, although with a large scatter between different simulations. In addition, the relative contributions of the three families of $\mathrm{BH}$ seed progenitors change and the total mass contributed by light $\mathrm{BH}$ progenitors is larger than that provided by medium-weight progenitors and comparable to that provided by heavy seeds.

\subsection{Effects of super-competitive accretion}

As anticipated in section 3.2 , we have also investigated the effects of the recently proposed SCA model (Chon \& Omukai 2020). In this model, SMSs leading to

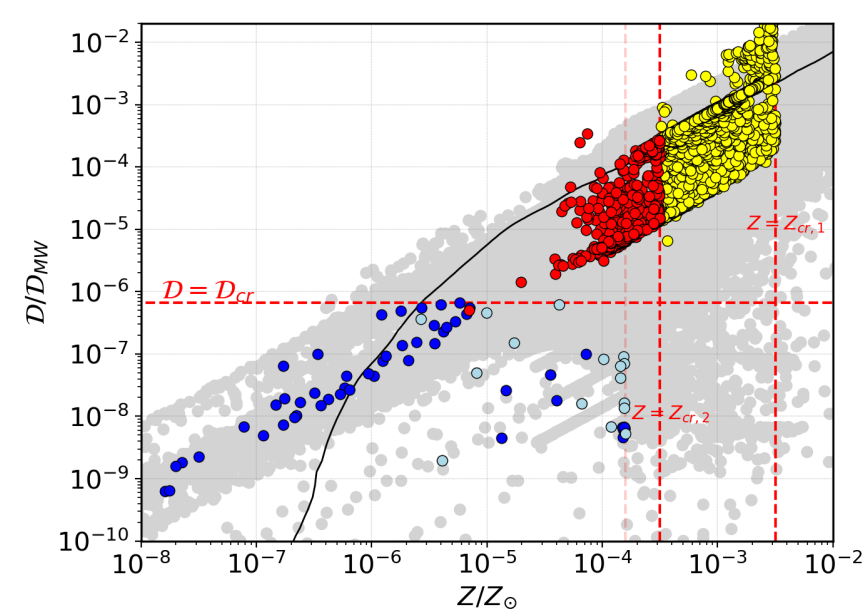

Figure 8. Same as Fig. 4 but for the set of parameters characterizing the SCA model and assuming $\boldsymbol{J}_{\mathrm{cr}}=300$ (model SCA300). Red dashed lines are the critical dust-to-gas ratio and metallicity thresholds adopted in the SCA model (see Table 2). The thin vertical line shows the metallicity threshold adopted in the reference model (see Fig. 4 and Table 2).
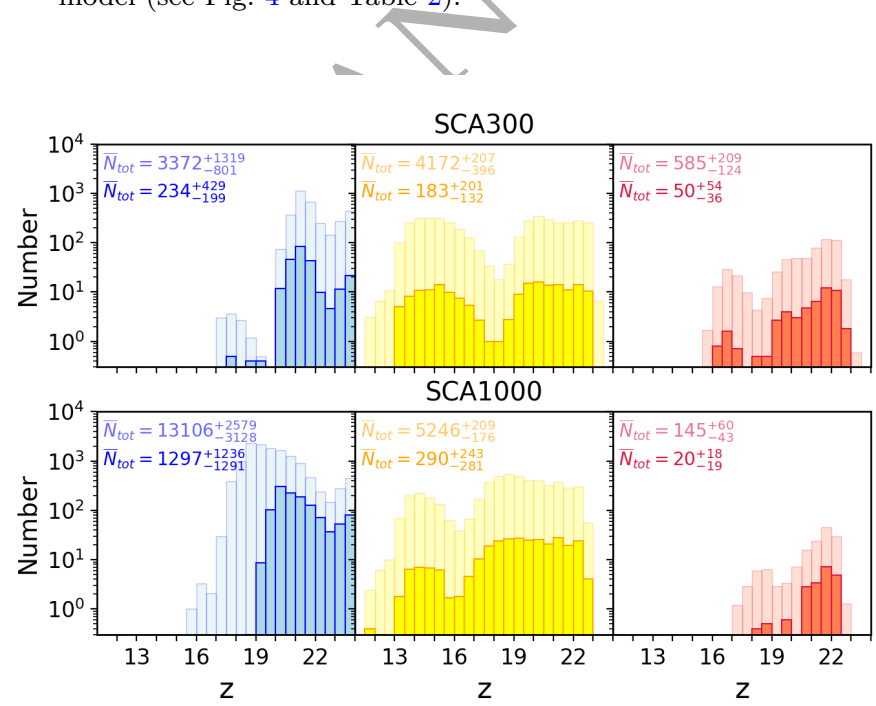

Figure 9. Same as Fig. 5 but for the set of parameters characterizing the SCA model and assuming $J_{\mathrm{cr}}=300$ (model SCA300, top panels) and $J_{\mathrm{cr}}=1000$ (model SCA1000, bottom panels).

medium-weight and heavy BH seeds may continue to form at higher metallicities than assumed in our reference model. In particular, we have run two additional sets of simulations, models SCA300 and SCA1000, where we have adopted the initial conditions of the SCA model summarized in Table 2 and two different values for $J_{\text {cr }}$ (300 and 1000, respectively). The results of these two additional models are illustrated in Figs. 8, 9, 10, and 11.

We first compare the results of model SCA300 with the corresponding reference run, R300. Note that, in order to ensure that the model reproduces the observed properties of our target quasar J1148 at $z=6.4$, we reduced the BH 


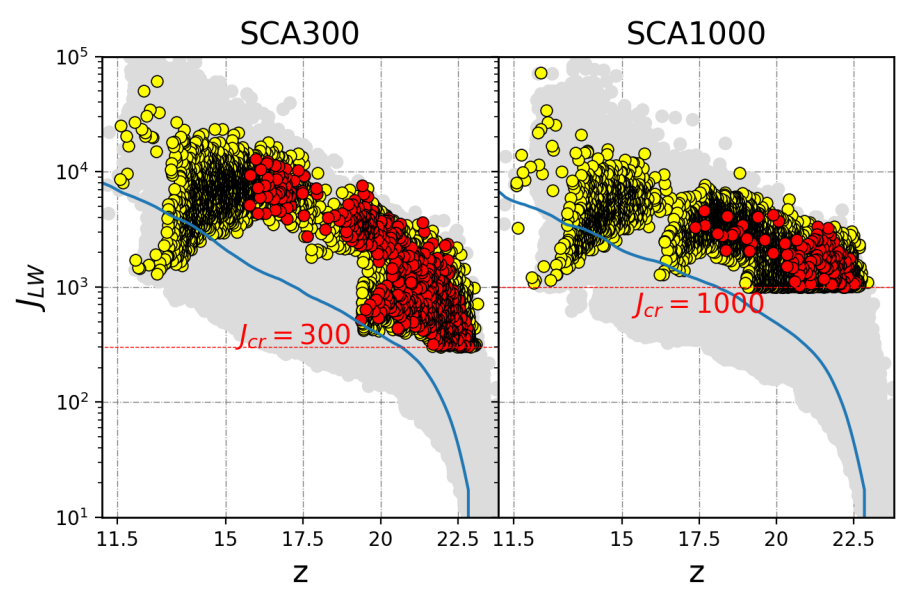

Figure 10. Same as Fig. 7 but for the set of parameters characterizing the SCA model and assuming $J_{\mathrm{cr}}=300$ (model SCA300, left panel) and $J_{\mathrm{cr}}=1000$ (model SCA1000, right panel).

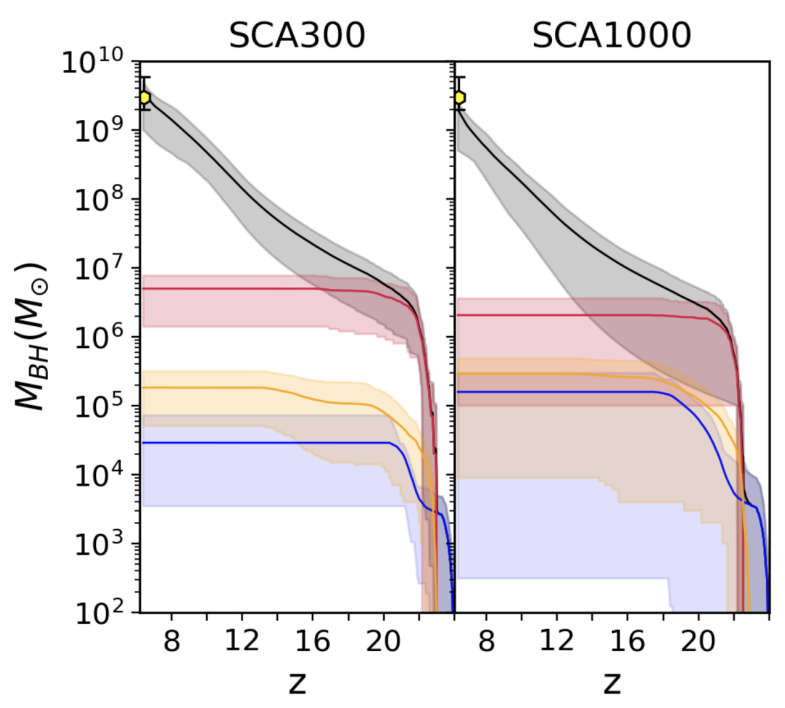

Figure 11. Same as Fig. 6 but for the set of parameters characterizing the SCA model and assuming $J_{\text {cr }}=300$ (model SCA300, left panel) and $J_{\mathrm{cr}}=1000$ (model SCA1000, right panel).

accretion efficiency, $\alpha_{\mathrm{BH}}$, from 150 (in the reference models) to 80 (in SCA models), as reported in Table 1.

Fig. 8 shows non-pristine formation sites of the different seed populations in one single simulation of the SCA300 model, to be compared with the R300 results in Fig. 4. The vertical and horizontal lines indicate the critical metallicity and dust-to-gas ratio thresholds adopted in the SCA model. As a consequence of the different conditions for their formation, both medium-weight and heavy seeds can form in a larger number of halos. Since in the SCA model dustdriven fragmentation does not prevent the formation of heavy seeds, some of the sites that were originally hosting middle-weight seeds in R300 can now lead to the formation of heavy seeds. This, however, does not reduce the number of medium-weight seeds as their formation can now occur in more metal enriched halos compared to the reference model.

Fig. 9 shows how the looser constraints on metallicity and dust-to-gas ratio in their birth clouds lead to a significant increase in the number of medium-weight and heavy $\mathrm{BH}$ seeds, compared to the reference model ( by a factor of $\sim 9$ and 53, respectively). This increase is particularly dramatic for medium-weight $\mathrm{BH}$ seeds, that become more numerous than light $\mathrm{BH}$ seeds in this model. The number of light $\mathrm{BH}$ seeds also increases ( by a factor of $\sim 1.8$ ), but this is an indirect effect of a decrease in the LW background intensity (compare the left panels of Figures 7 and 10). Indeed, a large fraction of halos that were assumed to form Pop II stars in model R300 host the formation of medium-weight BH seeds in model SCA300, leading to a decrease of the emitted LW radiation and hence of the global radiative feedback. This decrease favours the formation of Pop III stars and light BH seeds compared to model R300. At the same time, the decrease in $J_{\mathrm{LW}}$ (and its fluctuations) does not compromise the formation of medium-weight and heavy $\mathrm{BH}$ seeds, that can occur in gas with higher metallieity in the SCA model. Figures 9 and 10 show that in model SCA300 the formation of medium-weight and heavy seeds extends to lower redshift and is hosted in halos illuminated by lower $J_{\mathrm{LW}}$ compared to model R300.

Similar considerations apply if we restrict the analysis to $\mathrm{BH}$ seed progenitors: the darker coloured histograms in Fig. 9 show that they follow the same redshift distribution of their parent $\mathrm{BH}$ populations. Compared to model R300, the number of medium-weight and heavy $\mathrm{BH}$ seed progenitors formed in model SCA300 increases by a factor $\sim 3.3$ and 16.6, respectively.

The results change when the value of $J_{\mathrm{cr}}$ is increased to 1000, as in model SCA1000: compared to model SCA300, the total number of medium-weight seeds mildly increases, heavy seeds are a factor of $\sim 4$ less numerous and the number of light seeds increases by a factor of $\sim 3.8$. Hence, in this case, the milder radiative feedback favours Pop III star formation, and the associated metal enrichment disfavours the formation of heavy $\mathrm{BH}$ seeds. The $\mathrm{BH}$ seed progenitor populations are also affected, with heavy $\mathrm{BH}$ seeds being a factor $~ 2.5$ less numerous than in model SCA300, while medium-weight and light $\mathrm{BH}$ seed progenitors increasing by $\sim 1.6$ and $\sim 5.5$, respectively.

It is interesting to see how these differences affect the nuclear BH growth. A comparison between Figs. 6 and 11 shows that the more favourable conditions to heavy and medium-weight seeds formation in SCA models lead to the successful growth of $\mathrm{a} \sim 10^{9} \mathrm{M}_{\odot}$ SMBH at $z \sim 6$, independently of the adopted value of $J_{\mathrm{cr}}$. Despite the total $\mathrm{BH}$ mass contributed by medium-weight seeds is one order of magnitude higher than in the R300 and R1000 models, the results of SCA models confirm that the dominant mass contribution is provided by the less numerous but more massive heavy seeds.

\subsection{A composite picture: quality not quantity}

The analysis presented above highlights how sensitive the genealogy of $z \sim 6 \mathrm{SMBHs}$ is to $\mathrm{BH}$ seeds birth conditions. We find that the roots of the family tree are populated by light, medium-weight, and heavy $\mathrm{BH}$ ancestors, but their relative 


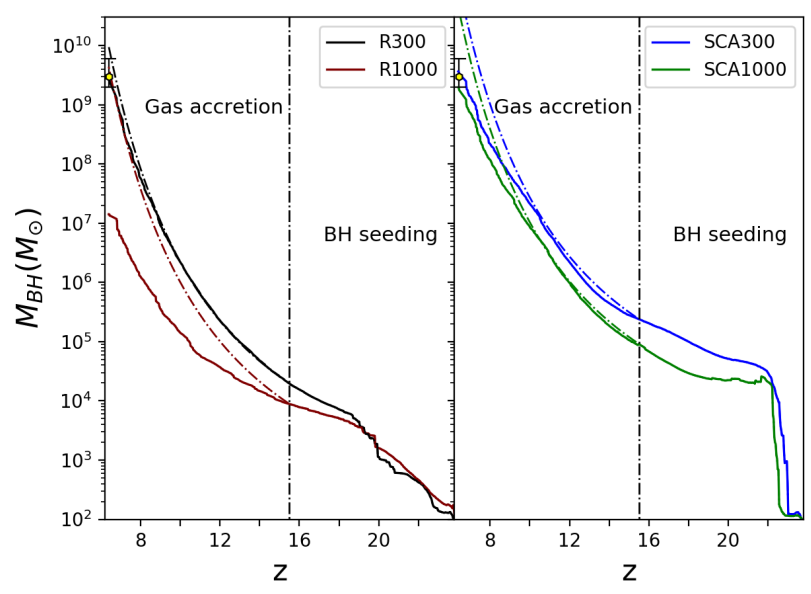

Figure 12. Left panel: evolution of the mean BH mass as a function of redshift in models R300 (black), R1000 (red). Each line is the average value among ten simulations. The vertical line indicates the epoch when seed formation terminates at $z \sim 15.5$. Below this redshift, nuclear BHs mostly grow by gas accretion. We compare the mean BH mass growth predicted by GQd models (solid lines) with the growth histories of $\mathrm{BHs}$ with a mass equal to the mean nuclear BH mass at $z \sim 15.5$ if these were assumed to grow uninterruptedly at the Eddington rate (dashed lines). Right panel: same as in the left panel but for models SCA300 (blue) and SCA1000 (green).

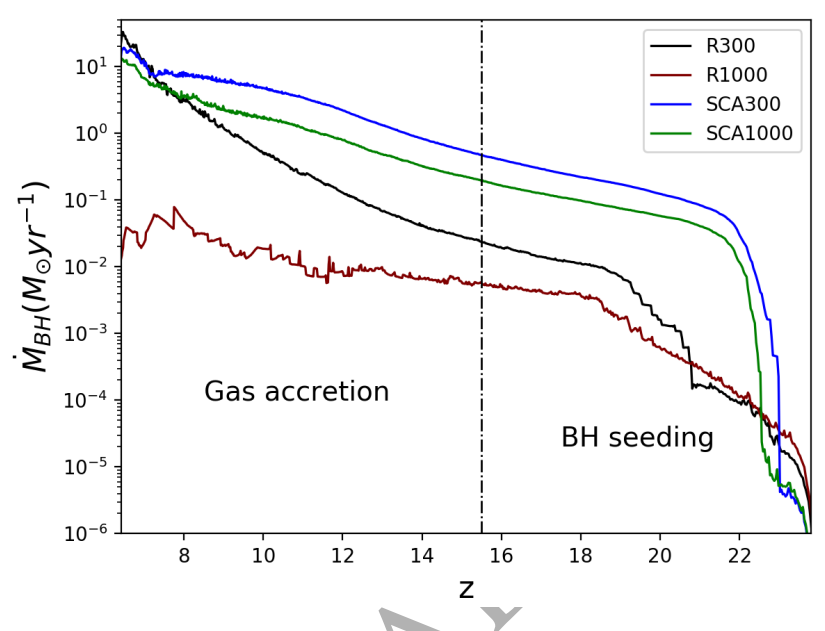

Figure 13. Evolution of the mean $\mathrm{BH}$ accretion rate as a function of redshift in models R300 (black), R1000 (red), SCA300 (blue), and SCA1000 (green). Each line is the average value among ten simulations. The vertical line indicates the epoch when seed formation terminates, at $z \sim 15.5$.

number and frequency is very sensitive to the combination of metallicity, dust-to-gas mass ratio, and illuminating LW flux that are assumed to provide the right conditions for their formation.

At early times, when $z \geq 16$, the evolution is dominated by $\mathrm{BH}$ seeding and the family tree that emerges can be densely populated, with a total mass in nuclear BHs that can be larger than $\sim 10^{6} \mathrm{M}_{\odot}$. However, we also find that a successful SMBH growth history relies on the quality of its $\mathrm{BH}$ seeds progenitors rather than on their quantity. Indeed, more than $99 \%$ of the final SMBH mass must come from gas accretion, that drives the evolution at later times, when $z<16$.

In our Eddington-limited accretion scenario, BHs grow at the Bondi rate that is very sensitive to $\mathrm{BH}$ mass (see Eq. 2.9). Hence, for the same total mass of $\mathrm{BH}$ seeds formed, family trees where the $\mathrm{BH}$ seeds mass distribution is more top heavy and the mean $\mathrm{BH}$ mass is larger are better equipped to grow to a $>10^{9} \mathrm{M}_{\odot} \mathrm{SMBH}$.

This is illustrated in Fig. 12, where we show the evolution of the mean $\mathrm{BH}$ mass as a function of redshift for the reference and SCA models, adopting two different values of the critical LW flux $\left(J_{\text {cr }}=300\right.$ and 1000).

As expected, at early times, during the $\mathrm{BH}$ seeding phase, the mean BH mass is strongly affected by the relative weight of different $\mathrm{BH}$ populations.

At the end of the BH seeding phase, the different seed $\mathrm{BH}$ mass distributions lead to mean $\mathrm{BH}$ masses that range between $\sim 10^{4} \mathrm{M}_{\odot}$ for model R1000 to $3 \times 10^{5} \mathrm{M}_{\odot}$ for model SCA300. During the early phase of the accretion-dominated evolution, a successful SMBH growth history requires rapid mass growth, close to the Eddington rate. To emphasize this point, for each model variant, the dashed lines show what would be the evolution if a single $\mathrm{BH}$ with a mass equal to the mean BH mass at $z \sim 16$ were assumed to evolve by growing uninterruptedly at the Eddington rate. The comparison between the solid and dashed lines, for each model, shows that with exception of model R1000, the mean BH mass initially grows very close or at the Eddington rate, and becomes sub-Eddington during the late phase of the evolution, at $z<10-12$

Fig. 13 shows the mean $\mathrm{BH}$ accretion rate as a function of redshift for the four models. During the BH seeding epoch, at $z \geq 15.5$, the accretion rates are affected by the evolving mass distribution of $\mathrm{BH}$ seeds progenitors. At all but the lowest redshifts, SCA models are characterized by the largest mean $\mathrm{BH}$ accretion rates, reflecting their larger mean BH mass. In model R300, the accretion rate increases more steeply than in SCA models, as a result of the larger gas mass in progenitor halos, that are less affected by AGN feedback. Finally, in model R1000 the mean accretion rate never exceeds $0.1 \mathrm{M}_{\odot} / \mathrm{yr}$ and shows a larger stochasticity, reflecting the smaller mean $\mathrm{BH}$ masses and the lower number of $\mathrm{BH}$ seeds in this model.

This result can be understood by looking at the mass distributions of $\mathrm{BH}$ seed progenitors that characterize the four models at the end of the $\mathrm{BH}$ seeding epoch. Fig. 14 shows the average distribution over 10 simulations at $z=15.5$. At the end of the BH seeding epoch, some BH progenitors have merged to form higher mass systems (i.e. the BHs in range $10^{4}<\mathrm{M}_{\mathrm{BH}} / \mathrm{M}_{\odot}<10^{5}$ or with $\left.\mathrm{M}_{\mathrm{BH}} \geq 10^{5} \mathrm{M}_{\odot}\right)$. The vertical lines in each panel indicate the mean $\mathrm{BH}$ mass, whereas the numbers refer to the total mass and number of BHs with $\mathrm{M}_{\mathrm{BH}} \geq 10^{5} \mathrm{M}_{\odot}$. These are the systems that drive the mass growth in the accretion-dominated phase at $z<15.5$. In models R300 and R1000, the number and total mass of BHs in this mass range is a factor of $\sim 10$ smaller than in models SCA300 and SCA1000, reflecting the difference in heavy $\mathrm{BH}$ seeds birth rate among these models. 
While in model R300 the enhanced accretion efficiency can counterbalance this large discrepancy, in R1000 the subsequent growth rate is too slow and leads to a final nuclear $\mathrm{BH}$ mass $\sim 2 \times 10^{7} \mathrm{M}_{\odot}$ at $z=6.4$, as shown by right panels of Figs. 6. To appreciate the large variety of possible growth histories among the different simulations, for each of the four models we report in the Appendix the $\mathrm{BH}$ mass evolution in each of the 10 runs, highlighting the contribution of light, medium-weight, and heavy $\mathrm{BH}$ seed progenitors.

\section{DISCUSSION}

Investigating the origin and growth history of $z \geq 6 \mathrm{SMBHs}$ is very challenging. While a number of studies have been devoted to explore BH seed formation (Valiante et al. 2017) and their subsequent evolution through mergers and gas accretion (Inayoshi et al. 2019), an ab-initio, self-consistent theory is still lacking.

Large-scale cosmological simulations such as MassiveBlack (Di Matteo et al. 2012), Illustris (Vogelsberger et al. 2014), EAGLE (Schaye et al. 2015), MassiveBlack-II (Khandai et al. 2015) and BlueTides (Feng et al. 2016) have been able to achieve good agreement with observations by implementing subgrid prescriptions for $\mathrm{BH}$ seed formation, which generally adopt a fixed BH seed mass that is planted in halos above a given mass threshold. In these approaches, both the $\mathrm{BH}$ seed mass and the minimum halo mass are free parameters of the simulation. More recently, attempts have been made to include in smaller-scale cosmological simulations or in zoom-in/constrained simulations more physically motivated prescriptions for $\mathrm{BH}$ seeding (Bellovary et al. 2011; Habouzit et al. 2017; Tremmel et al. 2017; Huang et al. 2020). Yet, the exploration of different seed physical parameters or their growth history is still too computationally expensive, despite attempts being made to avoid this limitation through post-processing methods (DeGraf \& Sijacki 2020).

Here we have followed a complementary approach and have used semi-analytical models to run independent evolutionary histories of a $10^{13} \mathrm{M}_{\odot}$ dark matter halo at $z=$ 6.4, following the evolution of its baryonic content since the onset of star and black hole formation at $z=30$. Building on a number of previous studies (Valiante et al. 2011, 2014, 2016b; Pezzulli et al. 2016; Pezzulli et al. 2017; Valiante et al. 2018a), here we have explored, for the first time, the incidence and relevance of three different BH seed populations for the early formation of SMBH. One important aspect of our investigation is that we do not restrict the analysis to a single BH/seed population. Rather, we populate the roots of SMBHs family trees with light, medium-weight, and heavy BH seeds depending on their specific birth conditions. In particular, here we have improved our previous model (Valiante et al. 2016b) by $(i)$ introducing the possibility to form medium-weight BH seeds and by (ii) implementing a new treatment of radiative, and chemical feedback that accounts for spatial fluctuations in the metal (dust) distribution and in the LW radiation field.

Despite these new features, our main conclusions agree with previous findings, demonstrating, for the first time, that the formation of medium-weight $\mathrm{BH}$ seeds does not qualitatively change the growth history of the first SMBHs. Indeed, similarly to Valiante et al. (2016b); Valiante et al. (2018a), we find that, if BHs are allowed to grow without exceeding their Eddington rate, the formation of the first SMBHs at $z>6$ relies on a small number of heavy $\mathrm{BH}$ seed progenitors. At the same time, we also find that medium-weight $\mathrm{BHs}$ are very common among the ancestors of $z=6.4 \mathrm{SMBHs}$, particularly in the SCA scenario proposed by Chon \& Omukai (2020). This is in broad agreement with the pioneering work of Devecchi \& Volonteri (2009); Devecchi et al. (2012), who also investigated when and where BHs could form as a result of runaway collisions of massive stars in dense nuclear star clusters. Here we have targeted our study to investigate the formation history of a prototypical quasar at $z=6.4$ instead of sampling a more representative cosmological volume. As a result, we find that despite medium-weight $\mathrm{BHs}$ always outnumber heavy BH seeds, their contribution to SMBH growth at $z>6.4$ is always subdominant with respect to that of heavy $\mathrm{BH}$ seeds.

One important caveat of our analysis concerns the assumed BH seed masses. While light BH seed masses are randomly sampled from an underlying Pop III stellar mass function and are therefore characterized by a mass distribution extending from a few tens to maximum $300 \mathrm{M}_{\odot}$, we have adopted a constant mass of $10^{3} \mathrm{M}_{\odot}$ and $10^{5} \mathrm{M}_{\odot}$ for medium-weight and heavy BH seeds, respectively. This is clearly an oversimplification, as it is expected that, depending on the physical conditions at place, medium-weight seeds may form with a range of masses extending from $\sim 300$ to $\sim 3000 \mathrm{M}_{\odot}$ (Devecchi et al. 2012) or $\sim 400$ to $\sim 1900 \mathrm{M}_{\odot}$ (Sakurai et al. 2017), or $\sim 100$ to $\sim 5000 \mathrm{M}_{\odot}$ (Reinoso et al. 2020). Similarly, heavy BH seeds are also expected to span a mass range that varies between $\sim 10^{3}$ to $[3-5] 10^{5} \mathrm{M}_{\odot}$ (Lodato \& Natarajan 2007), or $\sim 5 \times 10^{5}$ and $\sim 2 \times 10^{6} \mathrm{M}_{\odot}$ (Ferrara et al. 2014), or even to form in binary systems with masses of $10^{3}-10^{5} \mathrm{M}_{\odot}$ (Chon et al. 2018). In addition, these figures may be significantly altered in the SCA scenario (Chon \& Omukai 2020). Hence, the mass ranges of the three $\mathrm{BH}$ seed populations are not yet firmly assessed and may partly overlap, forming a continuum distribution from few tens to few $10^{5} \mathrm{M}_{\odot}$

Finally, it is worth mentioning that the formation of direct collapse BHs may be driven/triggered also by the combined effect of sub-critical LW radiation and dynamical heating, both contributing in suppressing fragmentation/star formation in rapidly growing pre-galactic halos, so that, in overdense regions, heavy seeds could be more common (larger number density) than previously expected (e.g. Wise et al. 2019b).

While a more refined description of the seed mass and its dependence on the birth conditions is deferred to a future study, here we anticipate that this may impact our results by changing the relative contribution of the three $\mathrm{BH}$ seeds populations to the mass growth of the final SMBH. More indirectly, it may also modify the baryonic evolution of their host galaxies and hence the relative frequency of mediumweight and heavy BH seeds that form at later times. Yet, our results suggest that, for each $\mathrm{SMBH}$ in place at $z=6.4$, a large number of $\mathrm{BH}$ seeds form at $z>15-16$ (see Figs. 5 and 9), only a small fraction of which are the progenitors of the final SMBH. Another caveat of our model is that we do not consider baryonic streaming motion (BSM), that may suppress star formation in less massive halos (typ- 


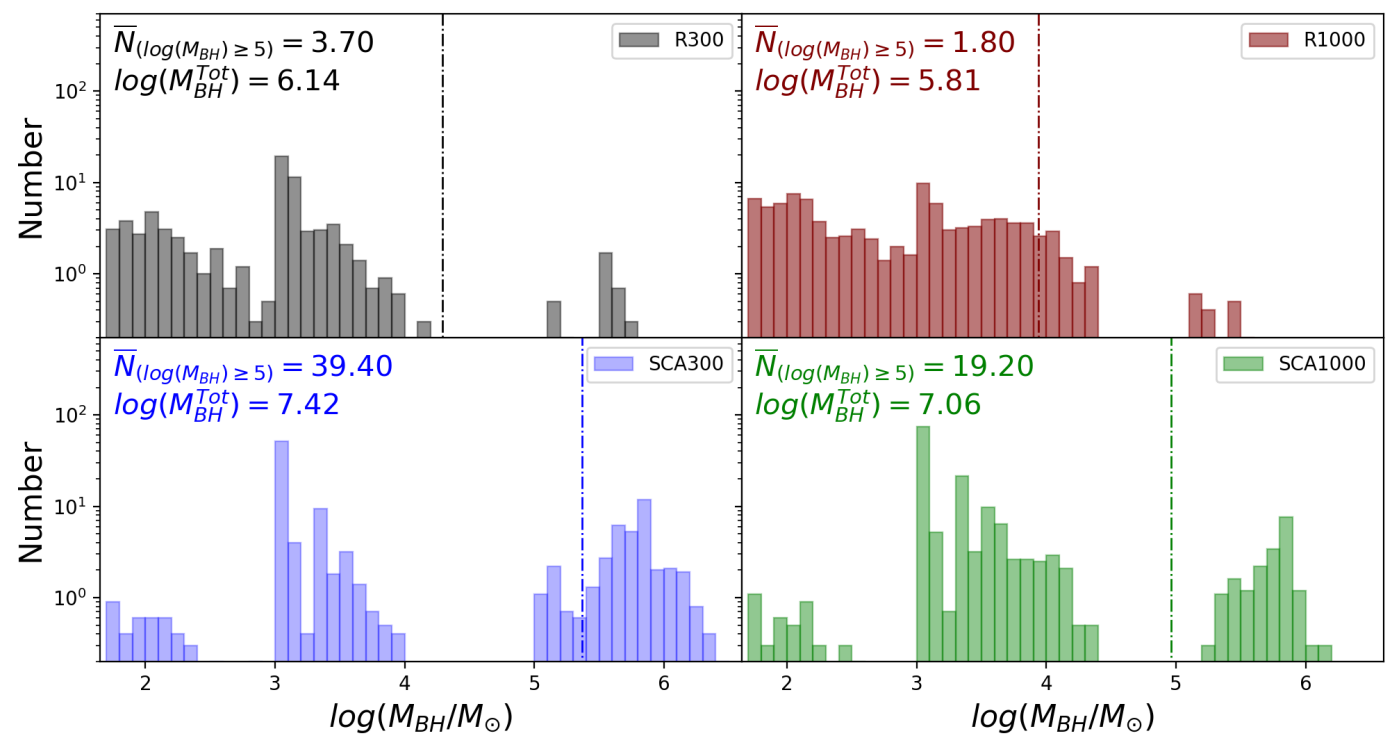

Figure 14. The mean BH mass function at $z=15.5$ averaged over 10 simulations of models R300 (top-left), R1000 (top right), SCA300 (bottom-left), and SCA1000 (bottom right). In each panel, the vertical line indicates the mean BH mass, whereas the numbers refer to the total mass and number of BHs with $M_{\mathrm{BH}} \geq 10^{5} \mathrm{M}_{\odot}$.

ically $T_{\text {vir }}<10^{4} \mathrm{~K}$ ), preventing gas collapse (Schauer et al. 2017; Inayoshi et al. 2018; Hirano et al. 2018). This limitation does not have a direct impact on the formation of heavy and medium-weight $\mathrm{BH}$ seeds, which form in larger, atomic cooling halos. However, the lack of star formation in minihalos may have an indirect effect because (i) the gas will remain chemically pristine until atomic-cooling halos form and (ii) the suppression of $\mathrm{H}_{2}$ cooling will be possible only by nearby star forming atomic cooling halos, in the so-called "syncronized-halo" model (Visbal et al. 2014). While it is not straightforward to assess the net effect of these two processes in our model, Schauer et al. (2017) argue that BSM may provide favourable conditions for the formation of heavy BH seeds. The minimum dark matter halo mass where gas cooling is suppressed by BSM increases $\left(T_{\mathrm{vir}} \sim 1-2 \cdot 10^{4} \mathrm{~K}\right)$ if the halo experiences a violent merger episode (Inayoshi et al. 2018). However these events are expected to berare, affecting less than $1 \%$ of the halos (see Fig. 2 in Inayoshi et al. 2018).

\section{SUMMARY AND CONCLUSIONS}

We have investigated the role of three $\mathrm{BH}$ seed populations in the formation history of $z>6 \mathrm{SMBHs}$. Building on previous studies, we have further extended our semi-analytical data-constrained model, GQd, by including a statistical description of the spatial fluctuations in metal and dust enrichment and in the intensity of the LW radiation field. For the first time, we have investigated the birth rate of light, medium-weight, and heavy BH seeds by selecting their formation sites according to physically motivated conditions on the metal and dust content as well as on the intensity of the illuminating LW radiation field (model R300). We find that:
- Inhomogeneous metal and dust enrichment and fluctuations in the LW radiation favour the formation of seeds and extends their birth epoch down to $z \sim 16$, when the filling factor of enriched regions becomes $Q \sim 1$;

- Light seeds are the first to form in pristine star-forming regions, and their formation is suppressed at $z \sim 20$ by radiative feedback that illuminates the metal-poor gas above the critical value, preventing $\mathrm{H}_{2}$-cooling. On average, $\sim 1831$ light seeds form in our reference model, but less than $\sim 13$ pér cent of these are true progenitors of the final $\mathrm{SMBH}$;

- Medium-weight and heavy BH seeds form at $16 \leq z \leq$ 23.5 when atomic cooling halos start to assemble. Mediumweight seeds form in metal-poor star-forming regions illuminated by a LW radiation $J_{\mathrm{cr}}<J_{\mathrm{LW}}<100 J_{\mathrm{cr}}$ from neighbouring systems, while heavy seeds mostly form in pristine regions when the illuminating LW intensity reaches values $10 J_{\mathrm{cr}}<J_{\mathrm{LW}}<100 J_{\mathrm{cr}}$. On average, 467 medium-weight and $\sim 11$ heavy seeds form in the reference model, $\sim 12-27 \%$ per cent of which are SMBH progenitors;

- Despite their smaller number, heavy BH seed progenitors provide the largest contribution to the SMBH mass growth, with a cumulative mass of $\sim 3 \times 10^{5} \mathrm{M}_{\odot}$ and triggering efficient gas accretion, that drives the mass growth at $z<16$ and leads to the formation of $\mathrm{a} \sim 3 \times 10^{9} \mathrm{M}_{\odot} \mathrm{SMBH}$ at $z=6.4$. For most of the time, the average gas accretion rate is very close to Eddington.

We have explored the sensitivity of the above results to variations of the adopted critical value of the LW flux by running a set of simulations with $J_{\mathrm{cr}}=1000$ (R1000). We find that:

- A larger $J_{\text {cr }}$ has the effect of reducing the strength of radiative feedback, favouring Pop III star formation and increasing the number of light seeds ( by a factor $\sim 5$ ). How- 
ever, this also causes a more efficient metal and dust enrichment, which limits the formation of medium-weight and heavy black hole seeds, decreasing their number by a factor $\sim 3.3-5.5$. A similar decrease ( by a factor of $\sim 2.2-3$ ) is also found in the number of medium-weight and heavy seed progenitor. This has a large impact on the formation of the SMBH, which on average reaches a mass of $\sim 2 \times 10^{7} \mathrm{M}_{\odot}$ by $z=6.4$, with a large dispersion of values among different evolutionary histories.

Finally, we have explored the possibility to form SMSs - leading to medium-weight and heavy BH seeds - at higher metallicity than assumed in our reference model, thanks to super-competitive accretion (Chon \& Omukai 2020). We find that:

- The looser constraints on metallicity and dust-to-gas ratio in the birth clouds predicted by this model (SCA300) lead to a significant increase in the total number of mediumweight (by a factor $\sim 9$ ) and heavy (by a factor $\sim 50$ ) $\mathrm{BH}$ seeds; the number of light seeds also increases (by a factor 2) due to the lower intensity of the LW background and the milder radiative feedback induced by a more significant contribution to the LW emissivity by accreting BHs, that are less efficient sources compared to Pop II stars. Heavy seeds dominate the mass growth, leading to the formation of $\mathrm{a} \sim 3 \times 10^{9} \mathrm{M}_{\odot} \mathrm{SMBH}$ at $z=6.4$, even when the critical intensity of the LW background is increased to $J_{\mathrm{cr}}=1000$ (SCA1000).

In the Eddington-limited accretion scenario that we have explored, we find that a successful SMBH growth history relies on the quality of its $\mathrm{BH}$ progenitors, rather than on their quantity: family trees whose roots are seeded by a sufficient number of heavy BH progenitors are better suited to grow $>10^{9} \mathrm{M}_{\odot}$ SMBHs by $z=6.4$.

Our study suggests that the genealogy of $z \sim 6 \mathrm{SMBHs}$ is characterized by a rich variety of $\mathrm{BH}$ progenitors, which represent only a small fraction $(<10-20 \%)$ of all the BHs that seed galaxies at $z>15.5$. While their mass distribution depends on the physical conditions (metallicity, dust-to-gas ratio, illuminating LW radiation) at their birth, these properties need to be explored in the attempt of making a census of the BH population at high- $z$. The present study is a first attempt to describe the richness of the $\mathrm{BH}$ landscape at cosmic dawn that may be explored by future electromagnetic and gravitational wave facilities.

\section{ACKNOWLEDGEMENTS}

We thank the Referee for her/his careful reading of the manuscript and insightful comments.

We acknowledge support from the Amaldi Research Center funded by the MIUR program "Dipartimento di Eccellenza "(CUP:B81I18001170001), from the INFN TEONGRAV specific initiative, and the networking support by the COST Action CA16104.

This work is partially supported by the National Science Foundation of China (11721303, 11991052, 11950410493), the National Key R\&D Program of China (2016YFA0400702).

$\mathrm{KO}$ and SC acknowledge financial support by the Grantsin-Aid for Basic Research by the Ministry of Education,
Science and Culture of Japan (SC:19J00324,KO:25287040, 17H01102, 17H02869).

F.S. thanks the University of Zürich for the kind support and hospitality and acknowledges the precious exchange of opinions and helpful advice of participants to the São Paulo School of advanced science on FIRST LIGHT and XXXI Canary Islands Winter School of Astrophysics.

\section{DATA AVAILABILITY}

The simulated data underlying this article will be shared on reasonable request to the corresponding author. The code GQd is not publicly available. The adopted approach and further possible improvements can be discussed with the corresponding author and the other code developers/users within the working group.

\section{REFERENCES}

Ade P. A., et al., 2014, Astronomy \& Astrophysies, 571, A16 Agarwal B., Khochfar S., Johnson J. L., Neistein E., Dalla Vecchia C., Livio M., 2012, Monthly Notices of the Royal Astronomical Society, 425, 2854

Agarwal B., Regan J., Klessen R. S., Downes T. P., Zackrisson E., 2017, MNRAS, 470,4034

Ahn K., Shapiro P. R., Iliev I. T., Mellema G., Pen U.-L., 2009, ApJ, 695, 1430

Amaro-Seoane P., Santamaria L., 2010, The Astrophysical Journal, 722, 1197

Argo M., Coppola J., Mezcua M., Earnshaw H., Roberts T., 2018, arXiv preprint arXiv:1812.05958

Bañados E., et al., 2016, ApJS, 227, 11

Baldassare V. F., Dickey C., Geha M., Reines A. E., 2020, The Astrophysical Journal Letters, 898, L3

Ballone A., Mapelli M., Pasquato M., 2018, Monthly Notices of the Royal Astronomical Society, 480, 4684

Bañados E., et al., 2018, Nature, 553, 473

Barack L., et al., 2019, Classical and quantum gravity, 36, 143001

Barrows R. S., Mezcua M., Comerford J. M., 2019, ApJ, 882, 181

Becerra F., Greif T. H., Springel V., Hernquist L. E., 2015, MNRAS, 446, 2380

Becerra F., Marinacci F., Bromm V., Hernquist L. E., 2018, MNRAS, 480, 5029

Bellovary J., Volonteri M., Governato F., Shen S., Quinn T., Wadsley J., 2011, ApJ, 742, 13

Bianchi S., Schneider R., Valiante R., 2009, in Henning T., Grün E., Steinacker J., eds, Astronomical Society of the Pacific Conference Series Vol. 414, Cosmic Dust - Near and Far. p. 65

Bondi H., 1952, Monthly Notices of the Royal Astronomical Society, 112, 195

Bondi H., Hoyle F., 1944, Monthly Notices of the Royal Astronomical Society, 104, 273

Bromm V., 2013, Reports on Progress in Physics, 76, 112901

Bromm V., Loeb A., 2003, The Astrophysical Journal, 596, 34

Bruzual G., Charlot S., 2003, MNRAS, 344, 1000

Callegari S., Mayer L., Kazantzidis S., Colpi M., Governato F., Quinn T., Wadsley J., 2009, ApJ, 696, L89

Callegari S., Kazantzidis S., Mayer L., Colpi M., Bellovary J. M., Quinn T., Wadsley J., 2011, ApJ, 729, 85

Capelo P. R., Volonteri M., Dotti M., Bellovary J. M., Mayer L., Governato F., 2015, MNRAS, 447, 2123

Chiaki G., Schneider R., Nozawa T., Omukai K., Limongi M., Yoshida N., Chieffi A., 2014, MNRAS, 439, 3121

Chiaki G., Marassi S., Nozawa T., Yoshida N., Schneider R., Omukai K., Limongi M., Chieffi A., 2015, MNRAS, 446, 2659 
Chon S., Omukai K., 2020, Supermassive Star Formation via Super Competitive Accretion in Slightly Metal-enriched Clouds (arXiv:2001.06491)

Chon S., Hirano S., Hosokawa T., Yoshida N., 2016, ApJ, 832, 134

Chon S., Hosokawa T., Yoshida N., 2018, MNRAS, 475, 4104

Clark P. C., Glover S. C., Klessen R. S., 2008, The Astrophysical Journal, 672, 757

Coleman Miller M., Hamilton D. P., 2002, Monthly Notices of the Royal Astronomical Society, 330, 232

Cubbon R. M., et al., 2007, European heart journal, 28, 540

Davies M. B., Miller M. C., Bellovary J. M., 2011, The Astrophysical Journal Letters, 740, L42

DeGraf C., Sijacki D., 2020, MNRAS, 491, 4973

Devecchi B., Volonteri M., 2009, The Astrophysical Journal, 694, 302

Devecchi B., Volonteri M., Rossi E. M., Colpi M., Portegies Zwart S., 2012, MNRAS, 421, 1465

Di Matteo T., Khandai N., DeGraf C., Feng Y., Croft R. A. C., Lopez J., Springel V., 2012, ApJ, 745, L29

Dijkstra M., Haiman Z., Mesinger A., Wyithe J. S. B., 2008, Monthly Notices of the Royal Astronomical Society, 391, 1961

Dijkstra M., Ferrara A., Mesinger A., 2014, Monthly Notices of the Royal Astronomical Society, 442, 2036

Dubois Y., Volonteri M., Silk J., Devriendt J., Slyz A., Teyssier R., 2015, MNRAS, 452, 1502

Fan X., Narayanan V. K., Strauss M. A., White R. L., Becker R. H., Pentericci L., Rix H.-W., 2002, AJ, 123, 1247

Fan X., et al., 2003, AJ, 125, 1649

Feng Y., Di-Matteo T., Croft R. A., Bird S., Battaglia N., Wilkins S., 2016, MNRAS, 455, 2778

Ferrara A., Salvadori S., Yue B., Schleicher D., 2014, MNRAS, 443, 2410

Frebel A., Norris J. E., 2015, ARA\&A, 53, 631

Fritze H., Wright S., Kilgard R., 2018, in American Astronomical Society Meeting Abstracts\# 231.

Graziani L., Schneider R., Ginolfi M., Hunt L. K., Maio U., Glatzle M., Ciardi B., 2019, arXiv e-prints, p. arXiv:1909.07388

Greene J. E., et al., 2010, ApJ, 721, 26

Greif T. H., 2015, Computational Astrophysics and Cosmology, 2,3

Gürkan M. A., Freitag M., Rasio F. A., 2004, The Astrophysical Journal, 604, 632

Habouzit M., Volonteri M., Latif M., Dubois Y., Peirani S., 2016a, MNRAS, 463, 529

Habouzit M., Volonteri M., Latif M., Dubois Y., Peirani S., 2016b, Monthly Notices of the Royal Astronomical Society, 463, 529

Habouzit M., Volonteri M., Dubois Y., 2017, MNRAS, 468, 3935

Heger A., Woosley S. E., 2002, ApJ, 567, 532

Hirano S., Hosokawa T., Yoshida N., Uméda H., Omukai K., Chiaki G., Yorke H. W., 2014, The Astrophysical Journal, 781, 60

Hirano S., Zhu N., Yoshida N., Spergel D., Yorke H. W., 2015, ApJ, 814, 18

Hirano S., Yoshida N., Sakurai Y., Fujii M. S., 2018, The Astrophysical Journal, 855, 17

Hosokawa T., Omukai K., Yoshida N., Yorke H. W., 2011, Science, 334,1250

Hosokawa T., Omukai K., Yorke H. W., 2012, ApJ, 756, 93

Hosokawa T., Hirano S., Kuiper R., Yorke H. W., Omukai K., Yoshida N., 2016, ApJ, 824, 119

Hoyle F., Lyttleton R. A., 1939, Proceedings of the Cambridge Philosophical Society, 35, 405

Huang K.-W., Ni Y., Feng Y., Di Matteo T., 2020, MNRAS, 496, 1

Iliev I. T., Scannapieco E., Martel H., Shapiro P. R., 2003, Monthly Notices of the Royal Astronomical Society, 341, 81

Inayoshi K., Tanaka T. L., 2015, Monthly Notices of the Royal Astronomical Society, 450, 4350
Inayoshi K., Omukai K., Tasker E., 2014, MNRAS, 445, L109

Inayoshi K., Haiman Z., Ostriker J. P., 2016, MNRAS, 459, 3738

Inayoshi K., Li M., Haiman Z., 2018, Monthly Notices of the Royal Astronomical Society, 479, 4017

Inayoshi K., Visbal E., Haiman Z., 2019, arXiv e-prints, p. arXiv:1911.05791

Jaacks J., Thompson R., Finkelstein S. L., Bromm V., 2018, MNRAS, 475, 4396

Jiang L., et al., 2016, ApJ, 833, 222

Johnson J. L., Dalla Vecchia C., Khochfar S., 2013, MNRAS, 428, 1857

Katz H., Sijacki D., Haehnelt M. G., 2015, MNRAS, 451, 2352

Khandai N., Di Matteo T., Croft R., Wilkins S., Feng Y., Tucker E., DeGraf C., Liu M.-S., 2015, MNRAS, 450, 1349

Kim S. S., Figer D. F., Morris M., 2004, The Astrophysical Journal Letters, 607, L123

Kremer K., et al., 2019, Physical Review D, 99, 063003

Lacey C., Cole S., 1993, Monthly Notices of the Royal Astronomical Society, 262, 627

Larson R. B., 1998, Monthly Notices of the Royal Astronomical Society, 301, 569

Latif M. A., Schleicher D. R. G., Schmidt W., Niemeyer J. C., 2013, MNRAS, 436, 2989

Latif M. A., Schleicher D. R. G., Hartwig T., 2016, MNRAS, 458,233

Lodato G., Natarajan P., 2007, MNRAS, 377, L64

Madau P., Ferrara A., Rees M. J., 2001, The Astrophysical Journal, 555, 92

Madau P., Haardt F., Dotti M., 2014, ApJ, 784, L38

Maio U., Khochfar S., Johnson J. L., Ciardi B., 2011, MNRAS, 414, 1145

Maio U., Borgani S., Ciardi B., Petkova M., 2019, Publ. Astron. Soc, Australia, 36, e020

Marassi S., Chiaki G., Schneider R., Limongi M., Omukai K., Nozawa T., Chieffi A., Yoshida N., 2014, ApJ, 794, 100

Matsukoba R., Takahashi S. Z., Sugimura K., Omukai K., 2019, Monthly Notices of the Royal Astronomical Society, 484, 2605

Matsuoka Y., et al., 2019, The Astrophysical Journal Letters, 872, $\mathrm{L} 2$

Mayer L., 2017, Nature Astronomy, 1, 0108

Mayer L., Bonoli S., 2019, Reports on Progress in Physics, 82,016901

Mayer L., Fiacconi D., Bonoli S., Quinn T., Roškar R., Shen S., Wadsley J., 2015, ApJ, 810, 51

Mezcua M., Civano F., Marchesi S., Suh H., Fabbiano G., Volonteri M., 2018, MNRAS, 478, 2576

Miller J. M., Fabian A., Miller M., 2004, The Astrophysical Journal Letters, 614, L117

Muratov A. L., Gnedin O. Y., Gnedin N. Y., Zemp M., 2013, ApJ, 773, 19

Nguyen D. D., et al., 2019, ApJ, 872, 104

Omukai K., 2001, ApJ, 546, 635

Omukai K., Nishi R., 1998, The Astrophysical Journal, 508, 141

Omukai K., Palla F., 2003, Astrophys. J., 589, 677

Omukai K., Tsuribe T., Schneider R., Ferrara A., 2005, The Astrophysical Journal, 626, 627

Omukai K., Schneider R., Haiman Z., 2008, The Astrophysical Journal, 686, 801

Omukai K., Hosokawa T., Yoshida N., 2010, The Astrophysical Journal, 722, 1793

Pallottini A., et al., 2015, MNRAS, 453, 2465

Petri A., Ferrara A., Salvaterra R., 2012, MNRAS, 422, 1690

Pezzulli E., Valiante R., Schneider R., 2016, MNRAS, 458, 3047

Pezzulli E., Volonteri M., Schneider R., Valiante R., 2017, Monthly Notices of the Royal Astronomical Society, 471, 589

Portegies Zwart S. F., McMillan S. L. W., 2002, ApJ, 576, 899

Raiteri C., Villata M., Navarro J., 1996, Memorie della Società astronomica italiana, 67, 817 
Regan J. A., Haehnelt M. G., 2009, MNRAS, 396, 343

Regan J. A., Johansson P. H., Wise J. H., 2014, ApJ, 795, 137

Regan J. A., Wise J. H., O'Shea B. W., Norman M. L., 2019, arXiv preprint arXiv:1908.02823

Reines A. E., Volonteri M., 2015, ApJ, 813, 82

Reinoso B., Schleicher D. R. G., Fellhauer M., Klessen R. S., Boekholt T. C. N., 2018, A\&A, 614, A14

Reinoso B., Schleicher D., Fellhauer M., Klessen R., Boekholt T., Vergara M., Alister Seguel P., 2019, Boletin de la Asociacion Argentina de Astronomia La Plata Argentina, 61, 154

Reinoso B., Schleicher D. R. G., Fellhauer M., Leigh N. W. C., Klessen R. S., 2020, arXiv e-prints, p. arXiv:2005.07807

Robert P. F., Murphy M. T., O'Meara J. M., Crighton N. H. M., Fumagalli M., 2019, MNRAS, 483, 2736

Safonova M., Shastri P., 2010, Astrophysics and Space Science, 325,47

Sakurai Y., Yoshida N., Fujii M. S., Hirano S., 2017, Monthly Notices of the Royal Astronomical Society, 472, 1677

Salvadori S., Tolstoy E., Ferrara A., Zaroubi S., 2014, MNRAS, 437, L26

Sarmento R., Scannapieco E., Côté B., 2019, ApJ, 871, 206

Sazonov S. Y., Ostriker J. P., Sunyaev R. A., 2004, MNRAS, 347,144

Schaerer D., 2002, A\&A, 382, 28

Schauer A. T. P., Regan J., Glover S. C. O., Klessen R. S., 2017, MNRAS, 471, 4878

Schaye J., et al., 2015, MNRAS, 446, 521

Schneider R., Omukai K., Bianchi S., Valiante R., 2012, MNRAS, 419, 1566

Sesana A., Haardt F., Madau P., Volonteri M., 2005, The Astrophysical Journal, 623, 23

Shakura N. I., 1973, Soviet Ast., 16, 756

Shang C., Bryan G. L., Haiman Z., 2010, Monthly Notices of the Royal Astronomical Society, 402, 1249

Shen R.-F., 2019, The Astrophysical Journal Letters, 871, L17

Sacdowski A., 2009, ApJS, 183, 171

Springel V., Di Matteo T., Hernquist L., 2005, Monthly Notices of the Royal Astronomical Society, 361, 776

Stone N. C., Küpper A. H., Ostriker J. P., 2017, Monthly Notices of the Royal Astronomical Society, 467, 4180

Sugimura K., Omukai K., Inoue A. K., 2014, Monthly Notices of the Royal Astronomical Society, 445, 544

Sugimura K., Matsumoto T., Hosokawa T., Hirano S., Omukai K., 2020, arXiv e-prints, p. arXiv:2002.00012

Tagawa H., Haiman Z., Kocsis B., 2020, The Astrophysical Journal, 892,36

Takekawa S., Oka T., Iwata Y., Tsujimoto S., Nomura M., 2019, The Astrophysical Journal Letters, 871, L1

Takeo E., Inayoshi K., Mineshige S., 2020, MNRAS,

Tornatore L., Ferrara A., Schneider R., 2007, Monthly Notices of the Royal Astronomical Society, 382, 945

Tremmel M., Karcher M., Governato F., Volonteri M., Quinn T. R., Pontzen A., Anderson L., Bellovary J., 2017, MNRAS, 470, 1121

Valiante R., Schneider R., Salvadori S., Bianchi S., 2011, Monthly Notices of the Royal Astronomical Society, 416, 1916

Valiante R., Schneider R., Salvadori S., Gallerani S., 2014, Monthly Notices of the Royal Astronomical Society, 444, 2442

Valiante R., Schneider R., Volonteri M., 2016a, Publications of the Astronomical Society of Australia, 33

Valiante R., Schneider R., Volonteri M., Omukai K., 2016b, Monthly Notices of the Royal Astronomical Society, 457, 3356

Valiante R., Agarwal B., Habouzit M., Pezzulli E., 2017, Publ. Astron. Soc. Australia, 34, e031

Valiante R., Schneider R., Graziani L., Zappacosta L., 2018a, MNRAS, 474, 3825

Valiante R., Schneider R., Zappacosta L., Graziani L., Pezzulli E., Volonteri M., 2018b, MNRAS, 476, 407
Valiante R., et al., 2020, MNRAS,

Van Wassenhove S., Capelo P. R., Volonteri M., Dotti M., Bellovary J. M., Mayer L., Governato F., 2014, Monthly Notices of the Royal Astronomical Society, 439, 474

Vanzella E., et al., 2020, arXiv e-prints, p. arXiv:2001.03619

Visbal E., Haiman Z., Bryan G. L., 2014, MNRAS, 442, L100

Vogelsberger M., et al., 2014, MNRAS, 444, 1518

Volonteri M., 2010, The Astronomy and Astrophysics Review, 18, 279

Walch S., Naab T., 2015, MNRAS, 451, 2757

Wang F., et al., 2021, A Luminous Quasar at Redshift 7.642 (arXiv:2101.03179)

Willott C. J., McLure R. J., Jarvis M. J., 2003, arXiv preprint astro-ph/0303062

Willott C. J., et al., 2010, AJ, 139, 906

Wise J. H., Turk M. J., Abel T., 2008, ApJ, 682, 745

Wise J. H., Turk M. J., Norman M. L., Abel T., 2012, ApJ, 745, 50

Wise J. H., Regan J. A., O'Shea B. W., Norman M.L., Downes T. P., Xu H., 2019a, Nature, 566, 85

Wise J. H., Regan J. A., O'Shea B. W., Norman M. L., Downes T. P., Xu H., 2019b, Nature, 566, 85

Wolcott-Green J., Haiman Z., Bryan G. L., 2017, MNRAS, 469, 3329

Wolcott-Green J., Haiman Z., Bryan G. L., 2020, Suppression of H2-cooling in protogalaxies aided by trapped Ly $\alpha$ cooling radiation (arXiv:2001.05498)

Woo J.-H., Cho H., Gallo E., Hodges-Kluck E., Le H. A. N., Shin J., Son D., Horst J. C., 2019, Nature Astronomy, 3, 755

Woosley S. E., Weaver T. A., 1995, ApJS, 101, 181

Woosley S. E., Heger A., Weaver T. A., 2002, Reviews of modern physics, 74, 1015

Wu X.-B., et al., 2015, Nature, 518, 512

Xu H., Norman M. L., O'Shea B. W., Wise J. H., 2016, ApJ, 823,140

Yang J., et al., 2020, ApJ, 897, L14

Yoshida N., Omukai K., Hernquist L., 2008, Science, 321, 669

Zhukovska S., Gail H. P., Trieloff M., 2008, A\&A, 479, 453

de Bennassuti M., Schneider R., Valiante R., Salvadori S., 2014 , Monthly Notices of the Royal Astronomical Society, 445, 3039 de Bennassuti M., Salvadori S., Schneider R., Valiante R., Omukai

K., 2017, Monthly Notices of the Royal Astronomical Society, 465,926

van den Hoek L. B., Groenewegen M. A. T., 1997, A\&AS, 123, 305

\section{APPENDIX A: BH MASS GROWTH}

In sections 4.4 and 4.6, we have discussed the evolution of the total mass of nuclear BHs as a function of redshift, highlighting the contribution of light, medium-weight, and heavy seeds in the R300, R1000, SCA300, and SCA1000 models. Figures 6 and 11 illustrate the trends obtained by averaging over 10 independent simulations of each model and the shaded regions represent the minimum and maximum value found at each $z$. The extent of these shaded regions indicates the large variety of individual evolutionary histories that we find, particularly in models R300 and R1000. In Figures A1, $\mathrm{A} 2, \mathrm{~A} 3$, and $\mathrm{A} 4$, we show the results obtained in each run of R300, R1000, SCA300, and SCA1000 models.

In model R300 (Figure A1) a similar evolution is found for the total BH mass (black lines) among all the 10 runs, with the exception of runs (2) and (3) where the final $\mathrm{BH}$ mass does not exceed $2 \times 10^{8} \mathrm{M}_{\odot}$, as a consequence of the lower black hole accretion rate.

The relative contribution of medium-weight and light 
BH seed progenitors can be very different, reflecting the impact of the cosmological evolution of the host galaxies on their birth conditions. The two populations provide almost equal contributions in some simulations, e.g. (9), or largely different ones in other, such as run (7), where only a few light $\mathrm{BH}$ seed progenitors form.

A completely different scenario is found in model R1000, where in all the runs the final SMBH is $<10^{8} M_{\odot}$ at $z=6.4$. This is a consequence of the smaller number (or absence, such as in run 3) of heavy BH seed progenitors formed and of their lower accretion rates. Indeed, a larger $J_{\text {cr }}$ leads to milder radiative feedback and to more efficient (Pop III) star formation, increasing the number of light $\mathrm{BH}$ seed progenitors and decreasing the amount of gas that feeds $\mathrm{BH}$ growth. In 5 out of 10 simulations, the total mass contributed light $\mathrm{BH}$ seeds is comparable or exceeds the one provided by heavy or medium-weight progenitors.

The 10 runs of the SCA300 (Figure A3) and SCA1000 (Figure A4) models show similar evolutionary histories, with significantly larger numbers of medium-weight and heavy seed progenitors, with respect to R300 and R1000. This reflects the looser constraints set by SCA models on the formation of SMSs. A SMBH $>5 \times 10^{8} \mathrm{M}_{\odot}$ is predicted at $z=6.4$ in all cases and (almost) independently of the adopted $J_{\text {cr }}$ threshold.

This paper has been typeset from a $\mathrm{T}_{\mathrm{E}} \mathrm{X} / \mathrm{LAT}_{\mathrm{E}} \mathrm{X}$ file prepared by the author. 

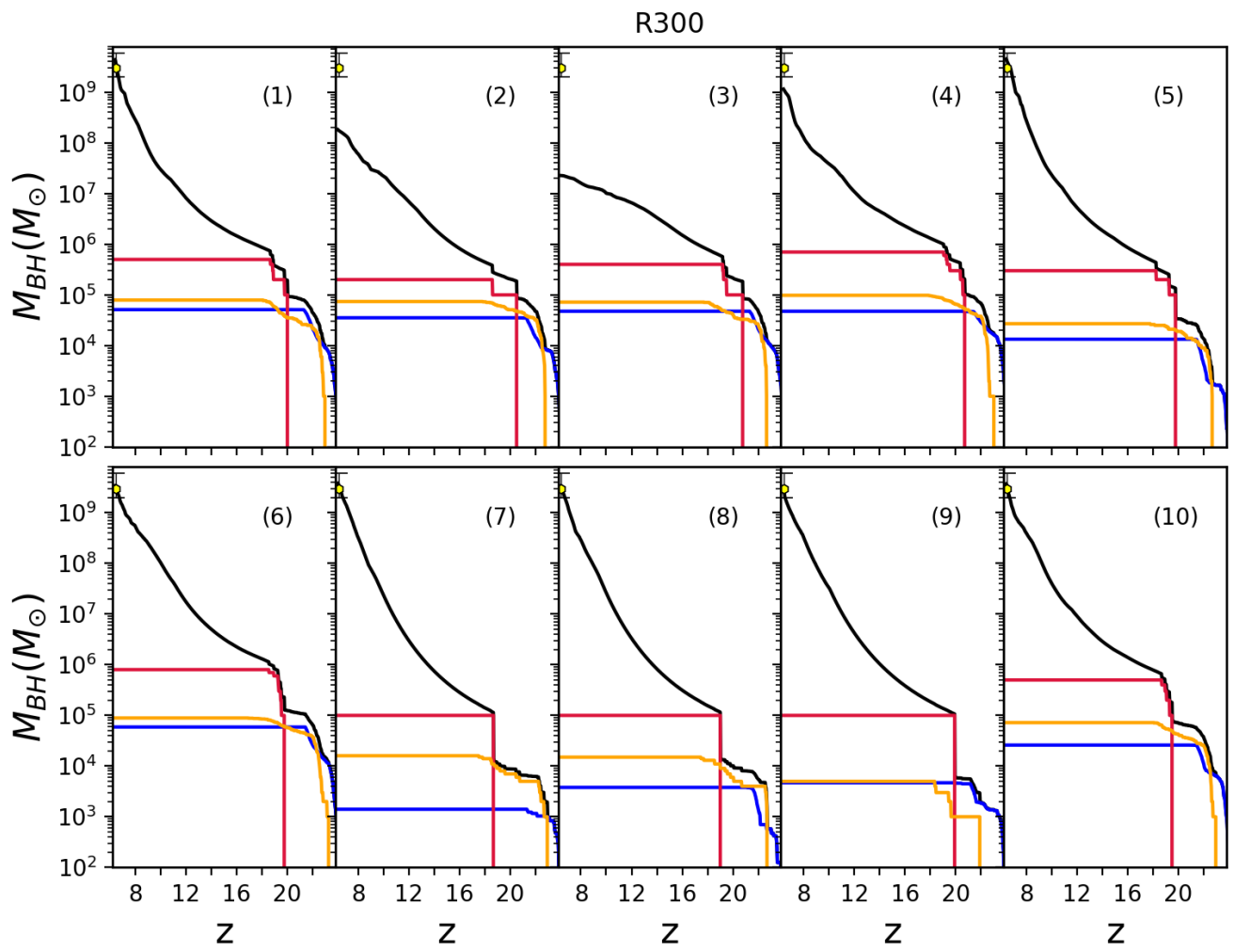

Figure A1. Evolution of the total mass of nuclear black holes as a function of redshift (black line) with the separate contributions of light (blue line), medium-weight (yellow line), and heavy BH seed (red line) progenitors. Each panel shows individual results over 10 simulations for R300 model.

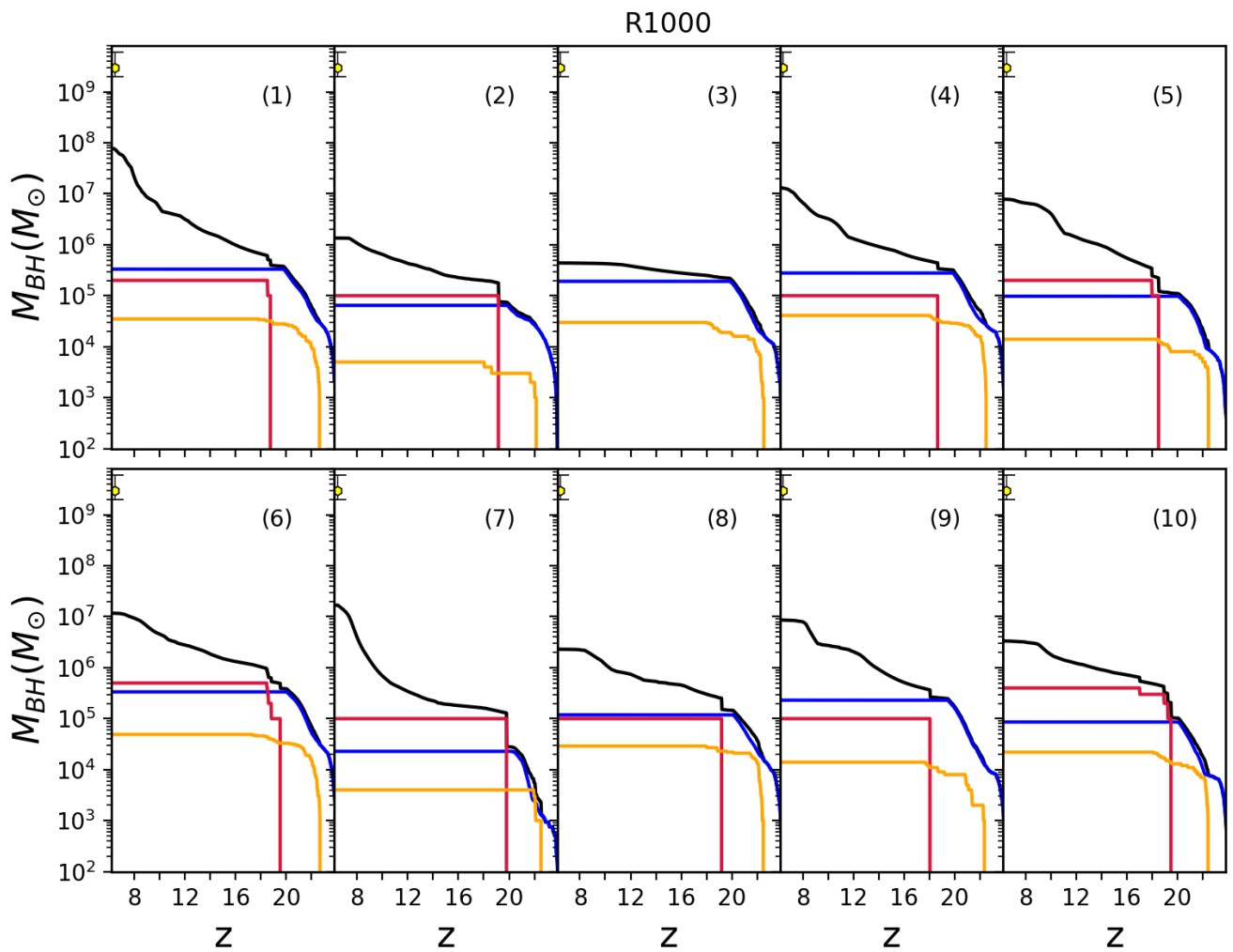

Figure A2. Same as Fig. A1 but for model R1000. 


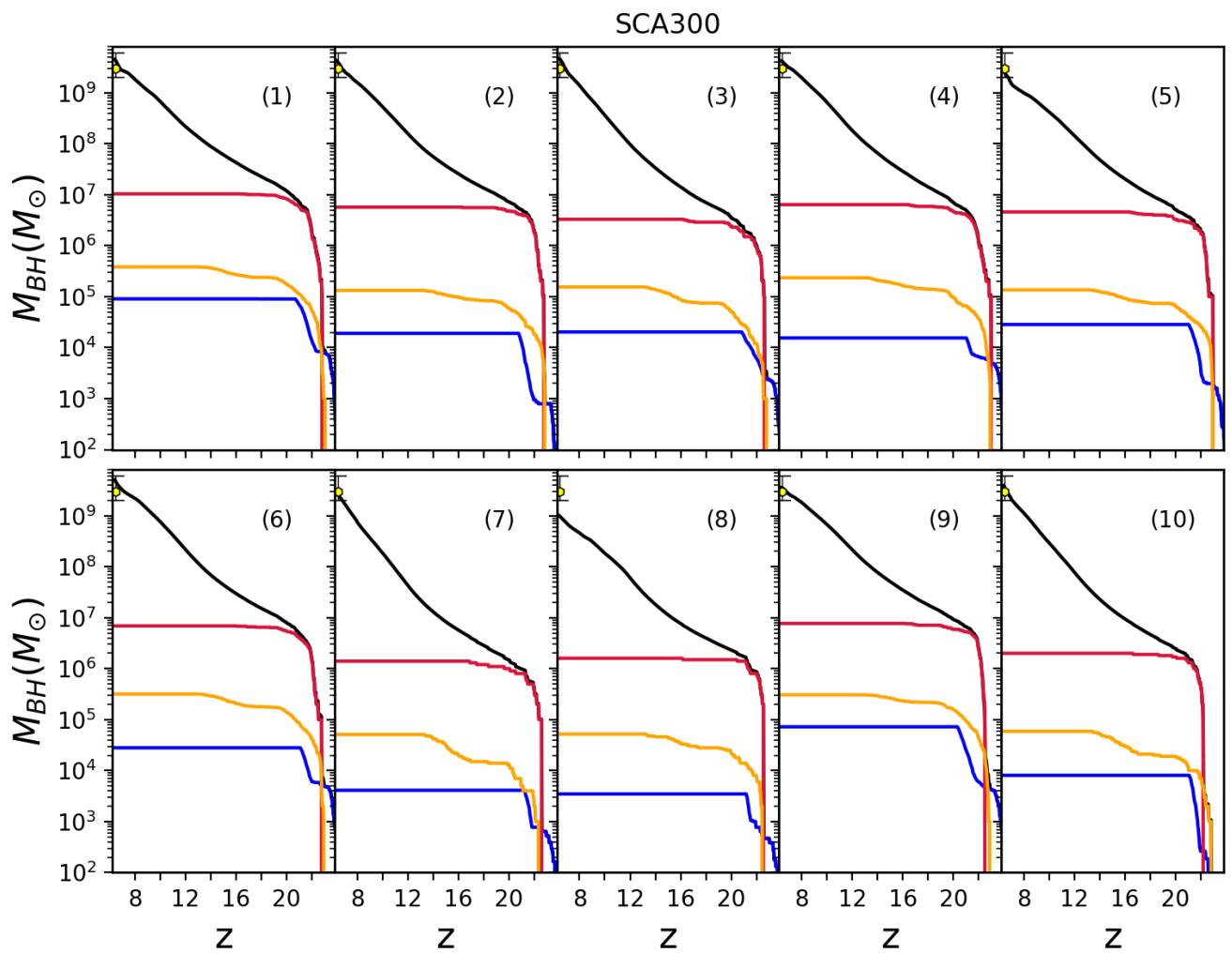

Figure A3. Same as Fig. A1 but for model SCA300.
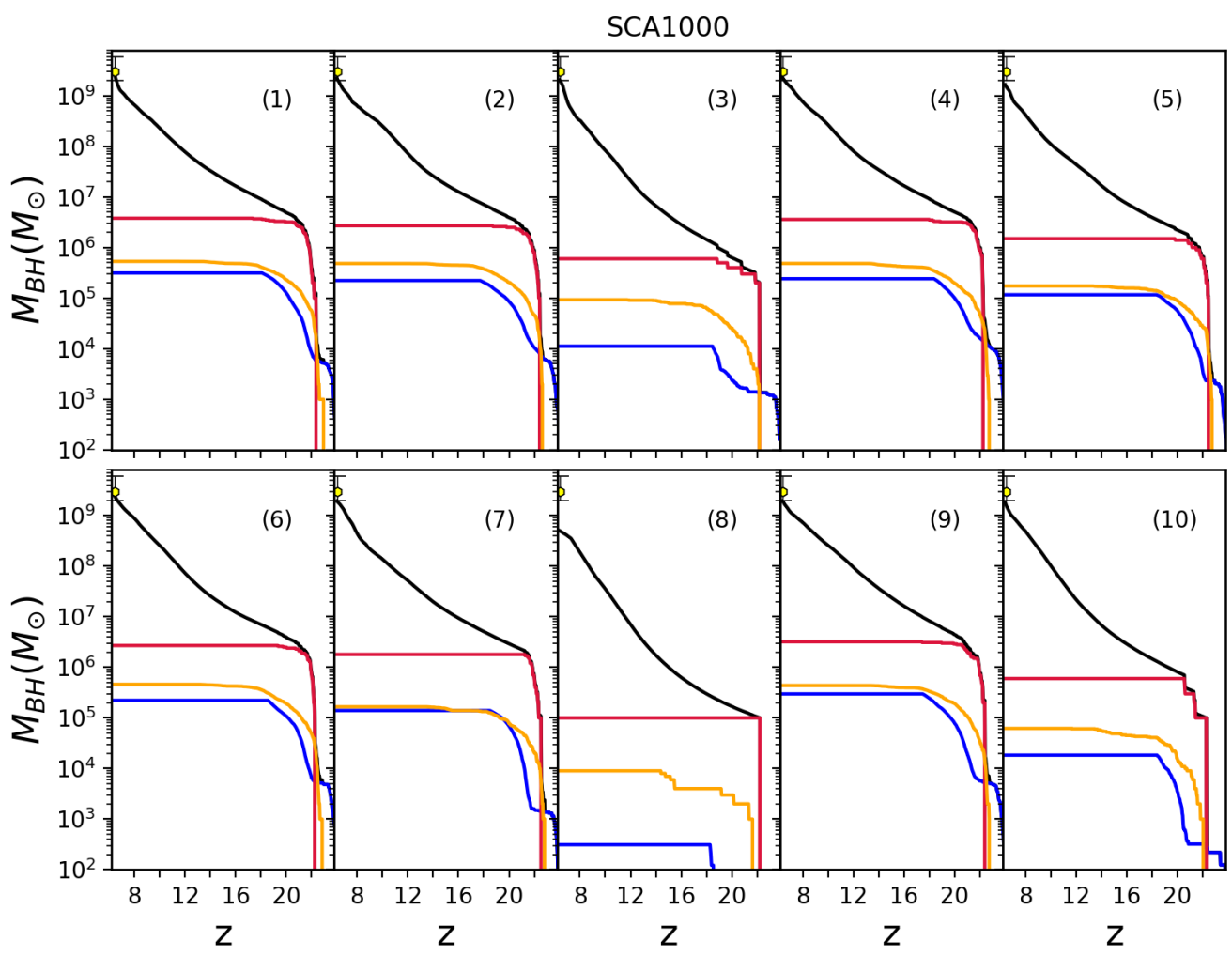

Figure A4. Same as Fig. A1 but for model SCA1000. 